\title{
Precision Measurements of Higgs Couplings: Implications for New Physics Scales
}

\author{
C. Englert, ${ }^{1}$ A. Freitas, ${ }^{2}$ M.M. Mühlleitner, ${ }^{3}$ T. Plehn, ${ }^{4}$ M. Rauch, ${ }^{3}$ M. Spira, ${ }^{5}$ and K. Walz ${ }^{3}$ \\ ${ }^{1}$ SUPA, School of Physics and Astronomy, University of Glasgow, United Kingdom \\ ${ }^{2}$ PITT-PACC, Department of Physics \& Astronomy, University of Pittsburgh, USA \\ ${ }^{3}$ Institut für Theoretische Physik, Karlsruhe Institute of Technology (KIT), Germany \\ ${ }^{4}$ Institut für Theoretische Physik, Universität Heidelberg, Germany \\ ${ }^{5}$ Paul Scherrer Institut, Villigen, Switzerland
}

The measured properties of the recently discovered Higgs boson are in good agreement with predictions from the Standard Model. However, small deviations in the Higgs couplings may manifest themselves once the currently large uncertainties will be improved as part of the LHC program and at a future Higgs factory. We review typical new physics scenarios that lead to observable modifications of the Higgs interactions. They can be divided into two broad categories: mixing effects as in portal models or extended Higgs sectors, and vertex loop effects from new matter or gauge fields. In each model we relate coupling deviations to their effective new physics scale. It turns out that with percent level precision the Higgs couplings will be sensitive to the multi-TeV regime.

\section{Contents}

\section{Introduction}

\section{Effective interactions}

2.1. Dimension-6 Lagrangian

Higgs self-interactions

Higgs-gauge boson interactions

Higgs-fermion interactions

2.2. Strongly interacting Higgs field

\section{Mixing effects}

3.3. Next-to-Minimal Supersymmetric Standard Model (NMSSM)

\section{Loop effects}

4.1. Simple examples

Heavy virtual bosons

Loop-induced decays

Vector-like leptons

4.2. $\tan \beta$-enhanced non-decoupling effects

\section{Summary and Conclusions}




\section{INTRODUCTION}

The recent discovery of the Higgs boson [1] at the LHC [2] can be considered as a triumph of quantum field theory in describing the fundamental interactions between elementary particles. The postulation of the Higgs boson defines the structure of the electroweak Standard Model (SM) and is the key ingredient to its renormalizability. While there might be good reasons to suspect that there exist intermediate new physics scales to account for dark matter, the quark flavor structure, neutrino masses, the baryon asymmetry of the Universe, or a full gauge coupling unification [3], the Standard Model is structurally complete. This means that we can, in principle, make statements about Lagrangians describing physics at the GUT-scale using renormalization group evolution from the weak-scale Lagrangian [4. In addition, the dominant production process as well as the most significant decay mode in the Higgs discovery are both induced by quantum effects. At the Born level the Higgs couples neither to gluons nor to photons, and the existence and the size of these loop-induced couplings is already a decisive test of the (effective) Standard Model [5].

With current data, all properties of the observed new state turn out to be in rough agreement with expectations of the Standard Model [6], but the experimental uncertainties are still large. A refinement of this coarse picture in the future may reveal deviations from the minimal SM scenario. The determination of zero-spin and positive parity [7, 8, required by isotropy of the vacuum, and the determination of the Higgs couplings to SM particles [9 12], gauge bosons and leptons/quarks, are the agents probing the Higgs mechanism sui generis for generating SM particle masses. The Higgs couplings are presently constrained at the level of several tens of percent, soon to be improved at the LHC [9, 10, 13] to about 20\%*. The high-luminosity run of the LHC (HL-LHC) will reduce the errors to about 10\%. Measurements at a future $e^{+} e^{-}$linear collider (LC) [10, 13, 14] can improve the accuracy to about 1\%, cf. Table I. Combining HL-LHC and HL-LC results will not give a significant improvement for most of the couplings. A notable exception is $h \gamma \gamma$, which is statistics limited even at the high-luminosity LC (HL-LC). The improved determination of the other couplings allows to better exploit the potential of the diphoton final state at the LHC, and, by chance, leads to the same precision for the $h \gamma \gamma$ and hgg couplings.

The interactions of the Higgs boson could deviate from their SM values if the Higgs mixes with other scalars, if it is a composite particle or a mixture between an elementary and composite state (partial compositeness),

\begin{tabular}{|l||c|c||c|c||c|}
\hline coupling & LHC & HL-LHC & LC & HL-LC & HL-LHC + HL-LC \\
\hline \hline$h W W$ & 0.09 & 0.08 & 0.011 & 0.006 & 0.005 \\
$h Z Z$ & 0.11 & 0.08 & 0.008 & 0.005 & 0.004 \\
$h t t$ & 0.15 & 0.12 & 0.040 & 0.017 & 0.015 \\
$h b b$ & 0.20 & 0.16 & 0.023 & 0.012 & 0.011 \\
$h \tau \tau$ & 0.11 & 0.09 & 0.033 & 0.017 & 0.015 \\
\hline$h \gamma \gamma$ & 0.20 & 0.15 & 0.083 & 0.035 & 0.024 \\
$h g g$ & 0.30 & 0.08 & 0.054 & 0.028 & 0.024 \\
\hline$h_{\text {invis }}$ & - & - & 0.008 & 0.004 & 0.004 \\
\hline
\end{tabular}

TABLE I: Expected accuracy at the $68 \%$ C.L. with which fundamental and derived Higgs couplings can be measured; the deviations are defined as $g=g_{S M}[1 \pm \Delta]$ compared to the Standard Model at the LHC/HL-LHC (luminosities 300 and $3000 \mathrm{fb}^{-1}$ ), LC/HL-LC (energies 250+500 GeV / 250+500 GeV+1 TeV and luminosities $250+500 \mathrm{fb}^{-1} /$ $1150+1600+2500 \mathrm{fb}^{-1}$ ), and in combined analyses of HL-LHC and HL-LC. For invisible Higgs decays we give the upper limit on the underlying couplings. Constraints on an invisible Higgs decay width involve model-specific assumptions at the LHC, see e.g. 15. Therefore, we allow for additional contributions to the total Higgs width only in the linear collider scenarios, where these can be constrained model-independently by exploiting the recoil measurement [14.

* Note that at the LHC we can only measure ratios of couplings without making model assumptions. 
or through loop contributions from other new particles. Thus, precision measurements of Higgs properties are sensitive to physics beyond the Standard Model, potentially residing at scales much higher than the Higgs vacuum expectation value (vev). Depending on the strength and type of coupling between the new physics and the Higgs boson, limits derived from Higgs data may exceed those from direct searches, electroweak precision measurements, or flavor physics. This way Higgs precision analyses open a unique window to new physics sectors that are not strongly constrained by existing results.

One way to study physics beyond the Standard Model (BSM) in terms of a well defined quantum field theory is given by the effective field theory approach. By assuming a few basic principles, like the field content and the gauge symmetries of the Standard Model, deviations from the Standard Model are parametrized by higherdimensional operators. While this approach allows us to study a large class of models it also has its limitations: for example, it cannot account for effects that arise from light particles, whose contributions may be enhanced in BSM models, or from Higgs decays into new non-SM particles. Therefore, to give a complete picture of BSM effects in the Higgs sectors we also study specific BSM models which capture such features.

In this paper we review characteristic scenarios to describe modified Higgs couplings from physics beyond the Standard Model, and illustrate their phenomenology with a few representative concrete models. In particular, we try to address the question what high-energy scales can be probed by precise measurements of Higgs couplings. While the answer to this question is necessarily model dependent, our aim is to work with a few typical scenarios and models that are archetypal examples for a much larger class of models. We start by introducing modified Higgs couplings in an effective theory philosophy in Section 2 . After that, we analyze the relationship between Higgs coupling deviations and the scale of new physics in two broad categories: modified Higgs properties through mixing effects (Section 3) and through loop effects (Section 4). In Section 5 , we evaluate and summarize the sensitivities to high scales in the different new physics scenarios.

\section{EFFECTIVE INTERACTIONS}

In the present article we will give a survey of typical scales of new physics beyond the Standard Model which can be probed in precision measurements of the Higgs couplings. Deviations from the SM values are predicted in many scenarios, of which a few representative examples will be described later in detail. Unless the underlying model violates the decoupling theorem [16], operator expansions [17, 18] suggest deviations of the order of

$$
g=g_{\mathrm{SM}}[1+\Delta]: \Delta=\mathcal{O}\left(v^{2} / \Lambda^{2}\right)
$$

with $v \approx 246 \mathrm{GeV}$ denoting the vacuum expectation value of the standard Higgs field and $\Lambda \gg v$ the characteristic scale of physics beyond the Standard Model. For typical examples of models which violate the decoupling theorem see Refs. [19, 20, and our discussion in Section 4.2 .

A theory with Standard Model operators but free Higgs couplings according to Eq.(2.1) is neither unitary nor electroweak renormalizable [21. However, it can be regarded as an effective theory which contains additional higher-dimensional operators suppressed by powers of $\Lambda$. The effective model can be thought of as emerging from a complete and UV-consistent fundamental theory with a decoupled heavy sector. One such completion could be a Two-Higgs-Doublet model $(2 \mathrm{HDM})$ 22, 23, with all heavy Higgs masses around the high scale $\Lambda$, well separated from the light Higgs mass (see end of this section).

According to Eq. 2.1) experimental accuracies of $\Delta=0.2$ down to 0.01 will give us sensitivity to scales of order $\Lambda \sim 550 \mathrm{GeV}$ up to $2.5 \mathrm{TeV}$. While the smaller of the two bounds is complementary to direct LHC searches, the larger of the two bounds generally exceeds the direct search range of LHC. Thus precision measurements in the Higgs sector may allow us to enter new physics territory.

A system in which the particles obey the symmetry structure of the Standard Model, but supplemented by 
interactions which are generated at high scales $\Lambda \gg v$, can be described by the effective interaction

$$
\mathcal{L}_{\text {eff }}=\sum_{D=2}^{6, \ldots} \frac{1}{\Lambda^{D-4}} \mathcal{L}_{D}
$$

Here $D$ characterizes the mass dimensions of the various terms of the effective Lagrangian. Higgs mass terms carry dimension $D=2$ and minimal interaction terms $D=4$. In this picture, large Higgs masses of $\mathcal{O}(\Lambda)$ can only be avoided either by fine-tuning operators of dimension $D=2$ or by introducing new symmetries, as for example in supersymmetric extensions or Little Higgs scenarios. For the analysis of any higher-dimensional system of the kind sketched in Eq. 2.2 it is crucial that one defines a complete operator basis and keeps in mind which set of operators a given coupling measurement corresponds to. As an example, the results of the Higgs couplings fit based on the $D \leq 4$ Lagrangian will change when we include a free Higgs coupling to photons or gluons at $D=6$. The measured central values and error bars, for example of the top Yukawa coupling, are critically affected by this change, so that every Higgs coupling extraction is defined in relation to a unique set of operators in the Lagrangian.

The effects of high-scale physics on the SM Higgs field and its interactions can be categorized in two classes: (i) mixing effects of the Higgs field with other high-mass scalar fields, and (ii) vertex effects modifying the couplings between the Higgs field and gauge bosons, quarks and leptons, and Higgs self-interactions.

(i) Mixing effects: The standard Higgs field may mix with other scalar fields. The operators describing the mixing effects carry mass dimension $D=4$ in the combined $\mathrm{SM} /$ new scalar system, reducing to $D=2$ in the effective SM Lagrangian after symmetry breaking. There, mixing reduces the mass of the Higgs boson by $\mathcal{O}\left(\eta^{2}\right)$, with $\eta$ denoting the coupling between the SM Higgs boson and the new scalars. The mixing also modifies the strength with which the field couples to SM particles. In basic portal models, in which the SM Higgs field is coupled with a hidden sector 24 27, the couplings are reduced universally. The decays of SM particles into states of the hidden sector demand proper control of invisible Higgs decays if this scenario should be described conclusively [28. A generic weakly interacting extension of the simplest Higgs sector includes a second Higgs doublet, as required in many models for physics beyond the Standard Model. Alternatively, the Higgs sector can be strongly interacting and connected to theories of extra dimensions by an AdS/CFT correspondence [26, 29 32. Finally, analyses in the decoupling regime 23] for large CP-odd pseudoscalar Higgs masses may open windows to areas not accessible in direct searches. It turns out that in this class energy scales with BSM physics can eventually be probed at the multi-TeV level.

(ii) Loop effects: Vertex corrections of Higgs couplings to SM particles can be generated by virtual contributions of new gauge bosons, scalars or fermions, colored or non-colored. Typical examples are predicted in a large variety of models, for example supersymmetry, strong dynamics, extra dimensions, see-saw models, or extended gauge groups. The vertex effects which are generated by the exchange of new heavy fields carry dimensions $D \geq 6$ for an $S U(2)$ doublet Higgs field. Such loop effects come with suppression factors $1 /\left(16 \pi^{2}\right)$ in addition to potentially small couplings between the Standard Model and the new fields. Thus, only new mass scales not in excess of about $M<v /(4 \pi \sqrt{\Delta}) \sim 200 \mathrm{GeV}$ can be probed, in most models much less than the direct search reach at the LHC. As a result, loop effects are less promising for exploring new physics scales indirectly.

Before we discuss the effects of specific modifications of the SM Higgs sector, we briefly review the approach taken in most of the recent Higgs coupling analyses [9 12, 33, 34]. In these approaches, one introduces a number of free couplings in the SM Lagrangian, corresponding to the number of independently measured production and decay channels. In the left panel of Fig. 1 we show such a coupling fit for the maximum number of currently accessible couplings as well as reduced sets, using the program SFitter [9, 19]. This provides a non-trivial test of the Standard Model, in which all Higgs couplings are predicted by the minimal realization of the Higgs mechanism. 
At the LHC, Higgs decays into gauge bosons are very well established through their characteristic finalstate signatures [2] and are the most precisely measured Higgs channels. Higgs decays to tau leptons can be identified in associated Higgs production with a hard jet or in weak boson fusion [35]37, while decays to bottom quarks can be measured in associated $W H$ and $Z H$ production [38, 39. From these channels, one can obtain information about the Higgs couplings to photons, $W / Z$-bosons, tau leptons and bottom quarks. However, because current $H \rightarrow b \bar{b}$ measurements are not sufficiently sensitive to probe Standard Model coupling strengths, the information on the bottom Yukawa coupling in the SFitter analysis [9] is dominated by the observed total Higgs production rate. Finally, the information on the top Yukawa coupling comes entirely from the effective Higgs-gluon and Higgs-photon couplings. This is why in Fig. 1 we can choose to either show $\Delta_{t}$ or $\Delta_{g}$ as measured couplings. In the future, we should be able to extract $t \bar{t} H$ production, for example with a Higgs decay into bottom jets or $W$ bosons [40]. Recently, some studies have suggested to extract the top Yukawa coupling from the effective Higgs-gluon coupling by resolving the top loop in the boosted Higgs regime [41].

When interpreting these Higgs coupling measurements we need to keep in mind that an arbitrary modification of these couplings violates the ultraviolet properties of the Standard Model, like renormalizability and unitarity. This problem can be solved if we consider the SM Lagrangian with free Higgs couplings as an effective theory, which at some energy scale is completed by a weakly interacting renormalizable field theory. The only condition on this ultraviolet completion is that its free parameters allow a free variation of all Higgs couplings in the Standard Model. As an example, we can interpret the current Higgs coupling measurements in terms of an aligned Two-Higgs-Doublet model, where the Yukawa couplings of the two Higgs doublets are proportional to each other in flavor space. For simplicity, we assume custodial symmetry $\left(\Delta_{Z}=\Delta_{W} \equiv \Delta_{V}<0\right)$, which can be broken through loop effects at the percent level [42. At tree level the aligned 2HDM has five free parameters, including the mass of the charged Higgs boson contributing to the effective Higgs-photon coupling. To eventually allow for a free Higgs-gluon coupling it would have to be supplemented for example by a top partner state. In the right panel of Fig. 1 we compare the extracted free Higgs couplings with the corresponding fit to the aligned $2 \mathrm{HDM}$ parameters, translated into the SM coupling deviations. We see that the central values as well as the error bars agree well between these two approaches. Slight deviations arise because the complete model can induce correlations between the couplings. If the aligned 2HDM is the true underlying model, additional constraints arise from non-standard Higgs searches and electroweak precision and flavor constraints. These have been ignored for the blue error bands, but are additionally taken into account for the cyan ones.

\subsection{Dimension-6 Lagrangian}

Assuming the new physics sector to be $S U(3) \times S U(2) \times U(1)$ gauge invariant, the leading effect of a heavy new physics sector on the SM Higgs field is described by effective $D=6$ operators [17, 18, 43, 45. For the introduction of scalar singlet states $D=5$ operators arise beyond the corresponding renormalizable dimension- 4 Lagrangian. Here we only consider an effective theory including the Higgs isodoublet $\phi$ supplemented by all other SM particles. The mass dimension of $\phi$ automatically induces a power counting in terms of a new energy scale $\Lambda$ [46] so that the effective Lagrangian can be written as

$$
\mathcal{L}_{\text {eff }}=\sum_{n} \frac{f_{n}}{\Lambda^{2}} \mathcal{O}_{n}
$$

with the couplings denoted by $f_{n}$. A list of the operators $\mathcal{O}_{n}$ relevant to Higgs phenomenology can be found in Table II. The complete, but not minimal list includes three types of operators: pure Higgs operators, Higgsgauge boson operators, and Higgs-fermion operators. These operators, written in terms of the $S U(2)_{L}$ doublet $\phi$, are defined in the linear representation of the Higgs and Goldstone fields. In this form the Lagrangian shows the full electroweak symmetry structure before electroweak symmetry breaking. The implicit physics assumption behind using this representation is that the particle discovered at the LHC is a Standard-Model-like 

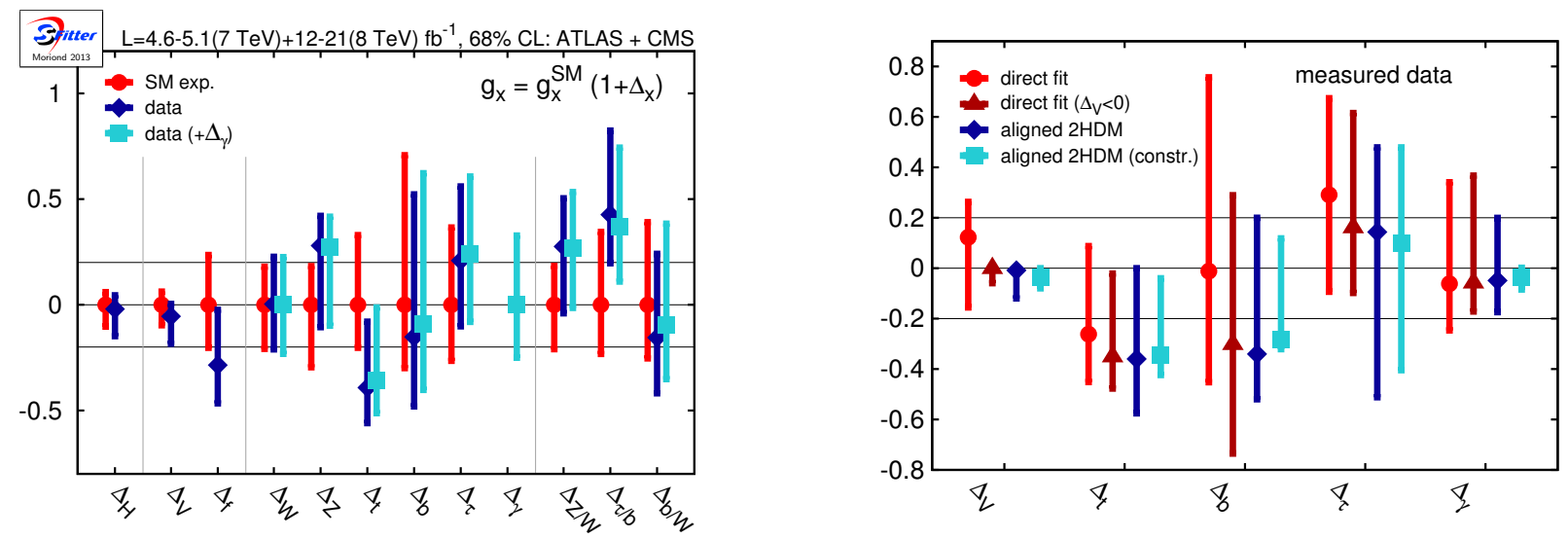

FIG. 1: Higgs coupling measurement based on all currently available ATLAS and CMS data. In the left panel we compare the SM expectation with a fit to the weak-scale Higgs Lagrangian with free couplings to the data, and either including a Higgs-photon coupling or not. In the last three columns we show errors on ratios of couplings, where, analogous to Eq.2.1], $\Delta$ parametrizes the deviation from the corresponding SM ratio. In the right panel we compare the fits to the weak-scale couplings with a fit to the aligned 2HDM in terms of the light Higgs couplings. Figures from Ref. [19. The only difference between the cyan results in the left panel and the lighter red ones in the right panel is that for the latter we set $\Delta_{W}=\Delta_{Z} \equiv \Delta_{V}$.

\begin{tabular}{|c|c|c|}
\hline Higgs-gluon & $\mathcal{O}_{G G}=\phi^{\dagger} \phi \operatorname{tr}\left\{G^{2}\right\}$ & SM Higgs phenomenology \\
\hline Higgs-vector boson (1) & $\begin{array}{l}\mathcal{O}_{\phi 1}=(D \phi)^{\dagger} \phi \phi^{\dagger}(D \phi) \\
\mathcal{O}_{\phi 4}=(D \phi)^{\dagger}(D \phi) \phi^{\dagger} \phi\end{array}$ & custodial symmetry violation \\
\hline Higgs-vector boson (2) & $\begin{array}{l}\mathcal{O}_{W W}=\phi^{\dagger} W^{2} \phi, \quad \mathcal{O}_{B B} \\
\mathcal{O}_{B W}=\phi^{\dagger} B W \phi \\
\mathcal{O}_{W}=(D \phi)^{\dagger} W(D \phi), \quad \mathcal{O}_{B}\end{array}$ & $\begin{array}{l}\text { SM Higgs decays } h \rightarrow \gamma \gamma, \gamma Z \\
\text { custodial symmetry violation }\end{array}$ \\
\hline Higgs-fermion (1) & \begin{tabular}{|l|}
$\mathcal{O}_{L R}=\left(\phi^{\dagger} \phi\right)(\bar{L} \phi R)$ \\
$\mathcal{O}_{L L 1}=\phi^{\dagger}(i \overleftrightarrow{D} \phi)(\bar{L} \gamma L), \quad \mathcal{O}_{R R 1}$ \\
$\mathcal{O}_{L L 3}=\phi^{\dagger}\left(i \overleftrightarrow{D^{a}} \phi\right)\left(\bar{L} \gamma \tau^{a} L\right)$ \\
\end{tabular} & $\begin{array}{l}\text { corrections to Yukawa couplings } \\
\text { neutral current contributions } \\
\text { neutral/charged current contributions }\end{array}$ \\
\hline Higgs-fermion (2) & $\mathcal{O}_{\phi B}=\phi \bar{L}(\sigma B) R, \quad \mathcal{O}_{\phi W}, \quad \mathcal{O}_{\phi G}$ & electric/magnetic moments \\
\hline Higgs self-coupling & $\begin{array}{l}\mathcal{O}_{\phi 2}=\frac{1}{2}\left|\partial\left(\phi^{\dagger} \phi\right)\right|^{2} \\
\mathcal{O}_{\phi 3}=\frac{1}{3}\left|\phi^{\dagger} \phi\right|^{3}\end{array}$ & $\begin{array}{l}\text { weak boson fusion, decays } h \rightarrow V V \\
\text { Higgs self-interactions }\end{array}$ \\
\hline
\end{tabular}

TABLE II: $D=6$ operators of an $S U(3) \times S U(2) \times U(1)$ invariant theory beyond the Standard Model involving the SM Higgs field. Notation: $\phi$ is the $S U(2)_{L}$ doublet; $W=W_{\mu \nu}=i \frac{g}{2} \sigma^{a} W_{\mu \nu}^{a}, B=B_{\mu \nu}=i \frac{g^{\prime}}{2} B_{\mu \nu}, G=G_{\mu \nu}=i \frac{g_{s}}{2} \lambda^{a} G_{\mu \nu}^{a}$ field strengths; $\partial$ space-time derivative, $D$ covariant derivative; $\phi^{\dagger} \stackrel{\leftrightarrow}{D} \phi=\phi^{\dagger} D \phi-(D \phi)^{\dagger} \phi ; L$ lepton/quark isodoublet, $R$ lepton/quark isosinglet; $1=$ isoscalar coupling, $3=$ isovector coupling; and $\gamma=\gamma_{\mu}, \sigma=\sigma_{\mu \nu}, \sigma^{a}$ Pauli matrices, $\lambda^{a}$ Gell-Mann matrices. Scale parameters $f_{G G} / \Lambda^{2}$ etc. define the impact of the operators. The conventions of Ref. [44 can be obtained by the identifications $\mathcal{O}_{L R} \rightarrow \mathcal{O}_{f \phi}, \mathcal{O}_{L L 1, R R 1} \rightarrow \mathcal{O}_{\phi f}^{(1)}, \mathcal{O}_{L L 3} \rightarrow \mathcal{O}_{\phi f}^{(3)}$.

Higgs boson, where deviations from the Standard Model case can be induced by mixing effects or an effective field theory description of an unknown ultraviolet completion.

Table II contains a rich chirality, isospin and flavor structure in higher-dimensional Higgs-fermion operators, scaled by $f_{G G} / \Lambda^{2}$ etc. This includes the usual scalar and vector currents, but also the dipole operators like $\mathcal{O}_{\phi B}$ etc. The operators in Table $\amalg$ are not independent but related by the [classical] equations of motion. Three equations connect the Higgs, gauge and fermion operators in a non-trivial manner [18, 44, in standard notation for the couplings and with the hypercharges for left-handed and right-handed fermions denoted by $Y_{L}$ 
and $Y_{R}$ :

$$
\begin{aligned}
\mathcal{O}_{\phi 4} & =-\mathcal{O}_{\phi 2}+\frac{1}{2} \sum_{\ell, q}\left(y \mathcal{O}_{L R}+\text { h.c. }\right)-\frac{1}{2} \frac{\partial V(h)}{\partial h} \\
\mathcal{O}_{B} & =-\frac{1}{2} \mathcal{O}_{B W}-\frac{1}{2} \mathcal{O}_{B B}-\frac{g^{\prime 2}}{2} \mathcal{O}_{\phi 1}+\frac{g^{\prime 2}}{4} \mathcal{O}_{\phi 2}-\frac{g^{\prime 2}}{4} \sum_{\ell, q}\left(Y_{L} \mathcal{O}_{L L 1}+Y_{R} \mathcal{O}_{R R}\right) \\
\mathcal{O}_{W} & =-\frac{1}{2} \mathcal{O}_{B W}-\frac{1}{2} \mathcal{O}_{W W}-\frac{g^{2}}{2} \mathcal{O}_{\phi 4}+\frac{g^{2}}{4} \mathcal{O}_{\phi 2}-\frac{g^{2}}{8} \sum_{\ell, q} \mathcal{O}_{L L 3} .
\end{aligned}
$$

The summed indices include the full generation structure of the Higgs-fermion operators. We are free to use these equations to eliminate three higher-dimensional Higgs operators of our choice. The discussion of the operators relevant for the purpose of this review becomes most transparent when we use these relations to directly eliminate $\mathcal{O}_{\phi 4}, \mathcal{O}_{B}$ and $\mathcal{O}_{W}$.

All operators given in Table II respect the gauge symmetry structure of the Standard Model. However, the Standard Model has additional (accidental) global symmetries motivated by experimental observations and with phenomenological implications. In the Higgs sector, custodial symmetry is broken at the loop level by the mass splitting of the fermion isodoublets and by gauging hypercharge as a subgroup of the bigger $S U(2)_{R}$ global symmetry of the SM Higgs sector, which guarantees $m_{W} / m_{Z}=\cos \theta_{W}$ for bare quantities. However, some of the $D=6$ operators lead to additional custodial breaking contributions, which can be described by the $S$ and $T$ parameters [47. At tree level one obtains [48],

$$
\alpha \Delta S=-e^{2} v^{2} \frac{f_{B W}}{\Lambda^{2}}, \quad \alpha \Delta T=-\frac{v^{2}}{2} \frac{f_{\phi 1}}{\Lambda^{2}} .
$$

Moreover, $\mathcal{O}_{B B}, \mathcal{O}_{W W}, \mathcal{O}_{B}, \mathcal{O}_{W}$ generate tree-level contributions to the extended set of oblique parameters $Y, W$ [49]. Compared to one-loop contributions in the Standard Model, which have been very successful in predicting the top and Higgs masses 42 these contributions are not necessarily small. These six operators, together with $\mathcal{O}_{L L 1}, \mathcal{O}_{L L 3}$ and $\mathcal{O}_{R R 1}$, are strongly constrained by $Z$-pole measurements and bounds on anomalous gauge boson interactions from $W^{+} W^{-}$production at LEP2 [12, 49]52. However, there are not enough independent electroweak precision observables to obtain separate bounds on all operators in this list. Therefore we here neglect $\mathcal{O}_{\phi 1}, \mathcal{O}_{B W}, \mathcal{O}_{L L 1}, \mathcal{O}_{L L 3}$ and $\mathcal{O}_{R R 1}$, but keep $\mathcal{O}_{B B}$ and $\mathcal{O}_{W W}$, which allow a significant deviation of the decay $h \rightarrow Z_{\gamma}$ from the SM prediction 53. This simple choice is adequate for current Higgs measurement uncertainties. In principle, however, one has to consider all dimension- 6 operators contributing to electroweak precision data at tree-level and carefully map out cancellations between them. Such cancellations are known to happen for example for vector resonances, kinetic mixing, or additional fermionic matter [20, 54, 57.

The dipole operators $\mathcal{O}_{\phi B}$ etc. are strongly constrained by measurements of the electric dipole moments and the anomalous magnetic moment, so that their contributions to the decay rates of the Higgs boson into fermion pairs can be neglected compared to $\mathcal{O}_{L R}$. For the third generation such dipole operators can eventually be tested in $t \bar{t} h$ and $b \bar{b} h$ production [58, which are production channels we do not consider here. Exploiting the equations of motion in Eq. 2.4., we are now left with the reduced operator basis

$$
\left\{\mathcal{O}_{G G}, \mathcal{O}_{W W}, \mathcal{O}_{B B} ; \mathcal{O}_{\phi 2}, \mathcal{O}_{\phi 3} ; \mathcal{O}_{L R}\right\}
$$

for the dimension-6 Higgs operators analysis.

The set of operators given in Eq.2.6. is a basis of the leading higher-dimensional operators given the symmetry structure of the ultraviolet completion of the Standard Model and our choice of using the equations of motion. However, there are many ways to reduce this dimension- 6 operator basis by making additional assumptions about the (experimentally unknown) model and its symmetry structure. From a phenomenological perspective such assumptions are not helpful. Instead, data from LHC and from a future $e^{+} e^{-}$linear collider should help us determine the structure of the Higgs sector based on the most general possible analysis. The only 
justification for additional simplifications can be fundamental shortcomings of the available data, for example a common lack of distinguishing power of collider searches. While we do not see how such an argument can be used in the gauge sector, we will resort to it for the Higgs couplings to fermions.

The dominant effect of higher-dimensional Higgs operators are modified relations between the dimension-4 Higgs potential $(\mu, \lambda)$ and the main observables $m_{h}$ and $v$. In addition, shifts in the Higgs wave function renormalization in general affect triple and quartic Higgs couplings, as well as an additional universal modification of the gauge boson-Higgs and fermion-Higgs couplings. The derivatives or momentum-dependent self interactions induced by $\mathcal{O}_{\phi 2}$ indicate strong self-interactions in the regime where the energy of the scattering process gets close to the suppression scale $\Lambda$. The price we pay for applying an effective field theory approach is that multiple Higgs couplings are generated with the same suppression factor $f_{\phi 2} / \Lambda^{2}$ [59]. Strong coupling effects for example from $\mathcal{O}_{\phi 2}$ can be observed experimentally as a significant rate enhancement in Higgs pair production [59 63] compared to single Higgs production at the LHC. In contrast, $\mathcal{O}_{\phi 3}$ will merely affect the value of the triple and quartic Higgs couplings at the LHC.

\section{Higgs self-interactions}

Since our main focus is not on the description of multi-Higgs interactions, we will adapt a canonical normalization of the Higgs kinetic term in the following. This implies a universal shift [44, 64]

$$
h \rightarrow\left[1-\frac{v^{2}}{4 \Lambda^{2}}\left(f_{\phi 1}+2 f_{\phi 2}+f_{\phi 4}\right)\right] h+\mathcal{O}\left(v^{4} / \Lambda^{4}\right),
$$

and the renormalization factors have to be included in the Higgs couplings. The link between the operators of Table II and observable Higgs interactions is given by the corresponding effective Lagrangian. Note that the induced shift in the Higgs mass term merely re-defines the bare Higgs mass, rather than inducing an observable effect.

We can write the Lagrangian containing solely pure Higgs interactions 64 to track the effects of the Higgs operators in Table II

$$
\begin{aligned}
\mathcal{L}_{\text {eff }}^{h}= & -\frac{m_{h}^{2}}{2 v}\left[\left(1-\frac{f_{\phi 124} v^{2}}{2 \Lambda^{2}}-\frac{2 f_{\phi 3} v^{4}}{3 \Lambda^{2} m_{h}^{2}}\right) h^{3}-\frac{2 f_{\phi 124} v^{2}}{\Lambda^{2} m_{h}^{2}} h \partial_{\mu} h \partial^{\mu} h\right] \\
& -\frac{m_{h}^{2}}{8 v^{2}}\left[\left(1-\frac{f_{\phi 124} v^{2}}{\Lambda^{2}}-\frac{4 f_{\phi 3} v^{4}}{\Lambda^{2} m_{h}^{2}}\right) h^{4}-\frac{4 f_{\phi 124} v^{2}}{\Lambda^{2} m_{h}^{2}} h^{2} \partial_{\mu} h \partial^{\mu} h\right],
\end{aligned}
$$

where $f_{\phi 124} \equiv \frac{1}{2} f_{\phi 1}+f_{\phi 2}+\frac{1}{2} f_{\phi 4}$. For completeness, we have included the contribution of $\mathcal{O}_{\phi 1}$, but will disregard it in the following, since it is strongly constrained by electroweak precision data. Also, at this point we have still retained the operator $\mathcal{O}_{\phi 4}$, which can be eliminated through the equations of motion in Eq.2.4. The corrections to the Higgs self-couplings appear in two distinct patterns. First, the Standard Model coupling strengths are modified by corrections of the form $v^{2} / \Lambda^{2}$ or $v^{4} /\left(m_{h}^{2} \Lambda^{2}\right)$, both of which are suppressed as long as $\Lambda \gg v$. Second, after Fourier transformation the last term in each line gives rise to modifications proportional to $p^{2} / \Lambda^{2}$. They are only small as long as the given observable probes small momentum scales $p \ll \Lambda$. Observables which probe a range of energies, such as longitudinal gauge boson scattering, will be dominated by physics at larger scales. If these are close to the cut-off scale we need to include an appropriate matching condition to the ultraviolet completion. However, at the LHC contributions from larger scales are usually suppressed by the parton densities, so that many observables are not sensitive to the particular structure of the ultraviolet completion. 
Higgs-gauge boson interactions

The same translation of the operators listed in Table $\Pi$ to an effective Lagrangian for the Higgs-gauge sector reads

$$
\begin{aligned}
\mathcal{L}_{\mathrm{eff}}^{h V} & =g_{h g g} h G_{\mu \nu}^{a} G^{a \mu \nu}+g_{h \gamma \gamma} h A_{\mu \nu} A^{\mu \nu} \\
& +g_{h Z \gamma}^{(1)} A_{\mu \nu} Z^{\mu} \partial^{\nu} h+g_{h Z \gamma}^{(2)} h A_{\mu \nu} Z^{\mu \nu} \\
& +g_{h Z Z}^{(1)} Z_{\mu \nu} Z^{\mu} \partial^{\nu} h+g_{h Z Z}^{(2)} h Z_{\mu \nu} Z^{\mu \nu}+g_{h Z Z}^{(m)} h Z_{\mu} Z^{\mu} \\
& +g_{h W W}^{(1)}\left(W_{\mu \nu}^{+} W^{-\mu} \partial^{\nu} h+\text { h.c. }\right)+g_{h W W}^{(2)} h W_{\mu \nu}^{+} W^{-\mu \nu}+g_{h W W}^{(m)} h W_{\mu}^{+} W^{-\mu}
\end{aligned}
$$

with $V_{\mu \nu}=D_{\mu} V_{\nu}-D_{\nu} V_{\mu}(V=B, W, G)$ supplemented by the corresponding rotations to the mass eigenstates $W^{ \pm}, Z, A$. The coupling strengths of the symmetric, higher-dimensional operators can be related to the Wilson coefficients of the effective Lagrangian of the broken theory as [44, 65]

$$
\begin{aligned}
g_{h g g} & =-\frac{g_{s}^{2} v}{2 \Lambda^{2}} f_{G G} & g_{h \gamma \gamma} & =-\frac{g^{2} v s_{W}^{2}}{2 \Lambda^{2}} \frac{f_{B B}+f_{W W}-f_{B W}}{2} \\
g_{h Z Z}^{(m)} & =\frac{g m_{Z}}{2 c_{W}}\left[1+\frac{v^{2}}{2 \Lambda^{2}}\left(f_{\phi 4}-f_{\phi 2}+\frac{f_{\phi 1}}{2}\right)\right] & g_{h W W}^{(m)} & =g m_{W}\left[1+\frac{v^{2}}{2 \Lambda^{2}}\left(f_{\phi 4}-f_{\phi 2}-\frac{f_{\phi 1}}{2}\right)\right] \\
g_{h Z Z}^{(1)} & =\frac{g^{2} v}{2 \Lambda^{2}} \frac{c_{W}^{2} f_{W}+s_{W}^{2} f_{B}}{2 c_{W}^{2}} & g_{h Z Z}^{(2)} & =-\frac{g^{2} v}{2 \Lambda^{2}} \frac{s_{W}^{4} f_{B B}+c_{W}^{4} f_{W W}+c_{W}^{2} s_{W}^{2} f_{B W}}{2 c_{W}^{2}} \\
g_{h W W}^{(1)} & =\frac{g^{2} v}{2 \Lambda^{2}} \frac{f_{W}}{2} & g_{h W W}^{(2)} & =-\frac{g^{2} v}{2 \Lambda^{2}} f_{W W} \\
g_{h Z \gamma}^{(1)} & =\frac{g^{2} v}{2 \Lambda^{2}} \frac{s_{W}\left(f_{W}-f_{B}\right)}{2 c_{W}} & g_{h Z \gamma}^{(2)} & =\frac{g^{2} v}{2 \Lambda^{2}} \frac{s_{W}\left[2 s_{W}^{2} f_{B B}-2 c_{W}^{2} f_{W W}+\left(c_{W}^{2}-s_{W}^{2}\right) f_{B W}\right]}{2 c_{W}},
\end{aligned}
$$

where $s_{W}$ and $c_{W}$ denote the sine and cosine of the weak mixing angle. As in Eq. 2.8), we also show how the contributions of $\mathcal{O}_{\phi 1}$ and $\mathcal{O}_{B W}$ enter in the formulae, but will continue to ignore these operators in the following. The effective scale parameter $\Lambda$ includes all possible couplings and loop factors of the kind $1 /\left(16 \pi^{2}\right)$. In Section 4.1 we will see that in specific models, where these additional factors are known, the bounds on the actual mass scale can be significantly weaker than in the general form of Eq. 2.9 due to loop suppression factors.

In these general expressions, Eqs.2.8 and 2.10, we have not yet made use of the equations of motion in Eq. 2.4 , because there is no general agreement which three operators to remove with their help. The couplings $g_{h V V}^{(m)}$ are linked to the heavy gauge boson masses and already exist in the renormalizable dimension-4 SM Lagrangian. To arrive at our basis of Eq. 2.6 we have to replace the coupling factors $f_{j}$ by a new set $f_{j}^{\prime}$, where

$$
f_{\phi 4}^{\prime}=f_{B}^{\prime}=f_{W}^{\prime}=0 .
$$

The remaining, finite coupling factors are shifted accordingly e.g. $f_{B B}^{\prime}=f_{B B}-f_{B} / 2 \ldots$, etc. The couplings $g_{h g g}$ and $g_{h \gamma \gamma}$ are generated by dimension- 6 operators, but play a special role both in experiment and in theory. First, in spite of being quantum effects they are the basis of the Higgs discovery at the LHC. Second, they are generated by SM states running in a closed loop and the corresponding operators are not suppressed by a heavy mass scale like $\Lambda=m_{t}$, see also Section 4.1. The Yukawa coupling between the Higgs boson and a massive chiral fermion in $f_{G G}$ circumvents the Appelquist-Carazzone decoupling theorem [16]. It leads to a scaling $f_{G G} \sim \Lambda^{2} / v^{2}$ which cancels the explicit $1 / \Lambda^{2}$ suppression and replaces it by $1 / v^{2}$. This non-decoupling behavior is the basis for the experimental exclusion of a chiral fourth generation [20, 66].

The alert reader might realize that modifying the $h W W$ and $h Z Z$ couplings as indicated in Eq.2.10 can lead to unitarity violation within the effective theory picture already for scales $p^{2}<\Lambda^{2}$. This modification in longitudinal gauge boson scattering will be compensated by new heavy scalar or vector resonances, which are 


\begin{tabular}{|l||c|c||c|c||c|}
\hline$\Lambda_{*}[\mathrm{TeV}]$ & LHC & HL-LHC & LC & HL-LC & HL-LHC + HL-LC \\
\hline \hline$h W W$ & 0.82 & 0.87 & 2.35 & 3.18 & 3.48 \\
$h Z Z$ & 0.74 & 0.87 & 2.75 & 3.48 & 3.89 \\
\hline$h t t$ & 0.45 & 0.50 & 0.87 & 1.34 & 1.42 \\
$h b b$ & 0.39 & 0.44 & 1.15 & 1.59 & 1.66 \\
$h \tau \tau$ & 0.52 & 0.58 & 0.96 & 1.34 & 1.42 \\
\hline$h g g$ & 0.55 & 1.07 & 1.30 & 1.80 & 1.95 \\
$h \gamma \gamma$ & 0.15 & 0.18 & 0.24 & 0.36 & 0.44 \\
\hline
\end{tabular}

TABLE III: Effective new physics scales $\Lambda_{*}$ extracted from the Higgs coupling measurements collected in Table [ The values for the loop-induced couplings to gluons and photons contain only the contribution of the contact terms, as the effects of the loop terms are already disentangled at the level of the input values $\Delta$.

integrated out and thus cannot be accounted for in the effective theory. Searches for such states have been described for example in Ref. 67] and can be considered independent of the Higgs measurements as long as the narrow width approximation is valid.

The labeling of the couplings in Eq. 2.10 correctly suggests that there are many ways to modify a Higgs coupling like $g_{h W W}$ through higher-dimensional operators. Such coupling shifts can arise from a non-standard renormalizable coupling as well as different dimension-6 operators. Based on rate measurements these effects cannot be distinguished. One way to gain some insight into the source of a possible deviation of LHC or linear collider measurements from the Standard Model prediction are additional constraints on the same set of dimension- 6 operators, for example from anomalous gauge couplings or electroweak precision data [44, 65, 68. Another way to separate different anomalous couplings are distributions, preferably in Higgs production processes which involve more particles than the Higgs boson. In that case the energy dependence or the Lorentz structure of an operator will be reflected in angular correlations or transverse momentum spectra of different particles produced [35, 46, 52, 68, 70.

\section{Higgs-fermion interactions}

In our illustrative analysis we are mainly concerned with collider measurements, where these couplings mediate flavor-diagonal fermionic decays assuming some kind of minimal flavor violation. For a review of flavorviolating objects we refer to Ref. [71. Moreover, we will only consider operators where current constraints from non-Higgs observables leave room for appreciable modifications of the Higgs branching ratios. Therefore, we limit ourselves to modifications of the fermion masses or Yukawa couplings,

$$
\mathcal{L}_{\text {eff }}^{h f f} \simeq-\frac{h}{\sqrt{2}} \sum_{b, t, \tau} \bar{L}\left(y-\frac{v^{2}}{2 \Lambda^{2}} f_{L R}^{\prime}\right) R+\text { h.c. }
$$

Again, the shifted coupling $f_{L R}^{\prime}$ differs from the general $f_{L R}$ in that now the equations of motion of Eq. 2.4 are used to define a minimal operator basis. The range of new physics scales that can be probed in the Higgs sector may be extracted from the parameters $\Delta$ collected in Table 1 which indicate potential deviations from the SM predictions of the Higgs couplings. According to the general analysis of independent operators introduced above, fermionic couplings and $g g, \gamma \gamma$ couplings prove particularly useful in this context while any deviations of $W W, Z Z$ couplings appear only on top of the large $h W W$ and $h Z Z$ tree-level couplings.

Now let us turn to the extraction of limits on the contributions of the $D=6$ operators. We again use SFitter [9] for this purpose. It is useful to define effective scales $\Lambda_{*}$ by factoring out from the operators some 
typical coefficients, like couplings of the kind $y, f_{X}^{\prime}$ and loop factors $1 /\left(16 \pi^{2}\right)$. In detail, we replace

$$
\begin{aligned}
& \text { fermions : } \quad \Delta_{f}=-\frac{v^{2}}{2 \Lambda^{2}} \frac{f_{L R}^{\prime}}{y} \quad \rightarrow \frac{v^{2}}{2 \Lambda_{*}^{2}[f]} \\
& W W, Z Z: \quad \Delta_{V}=-\frac{v^{2}}{2 \Lambda^{2}} f_{\phi 2}^{\prime} \quad \rightarrow 2 \frac{v^{2}}{2 \Lambda_{*}^{2}\left[V_{m}\right]} \\
& g g: \quad \Delta_{g}=-\frac{v^{2}}{2 \Lambda^{2}} \frac{4 \cdot 16 \pi^{2}}{\zeta_{g}} f_{G G}^{\prime} \quad \rightarrow \quad \frac{4}{\zeta_{g}} \frac{v^{2}}{2 \Lambda_{*}^{2}[G G]} \\
& \gamma \gamma: \quad \Delta_{\gamma}=-\frac{v^{2}}{2 \Lambda^{2}} \frac{2 \cdot 16 \pi^{2}}{\zeta_{\gamma}} \frac{f_{B B}^{\prime}+f_{W W}^{\prime}}{2} \rightarrow \frac{1}{\zeta_{\gamma}} \frac{v^{2}}{2 \Lambda_{*}^{2}[W W / B B]},
\end{aligned}
$$

where $G G$ denotes the gluonic contact term. The factors $\zeta_{g}=A_{1 / 2}\left(4 m_{t}^{2} / m_{h}^{2}\right) \simeq 4 / 3$ and $\zeta_{\gamma}=$ $(4 / 3) A_{1 / 2}\left(4 m_{t}^{2} / m_{h}^{2}\right)+A_{1}\left(4 m_{W}^{2} / m_{h}^{2}\right) \simeq-6.5$ account for the total SM loop amplitude, see Eqs.4.3) and (4.4). In the effective Higgs-gluon and Higgs-photon couplings the input values $\Delta$ already separate the contact terms from the loop terms, induced by modified $h t t$ and $h W W$ couplings. Therefore, we can directly identify $\Delta_{g, \gamma}$ with the corresponding contact terms without evaluating loop and contact terms individually. While we only show the contribution of top and $W$ loops in the formulae above, in the SFitter analysis all loop contributions are properly taken into account. The projected limits on the $\Lambda_{*}$ parameters as defined above are collected in Table III and Fig. 2 .

As we can see, the effective new physics scales that can be probed in the Higgs sector extend to a range from several hundred $\mathrm{GeV}$ to maximum values beyond a $\mathrm{TeV}$. However, bounds on new particle masses exchanged at the Higgs vertex may be reduced significantly by small couplings $M \sim \Lambda_{*} \sqrt{g^{2} / 16 \pi^{2}}$ as shown later in this section. Thus, it depends on the specific model to what extent precision Higgs analyses may explore high-mass domains in new physics scenarios beyond direct searches at high-energy colliders.

\subsection{Strongly interacting Higgs field}

While originally light Higgs bosons were foreign to concepts of strong electroweak symmetry breaking, the continuing support for light Higgs bosons by electroweak precision analyses 42 ] and finally the LHC discovery of a light, narrow single Higgs boson [2] suggested concepts within which a single light state is embedded in a heavy strongly interacting sector.

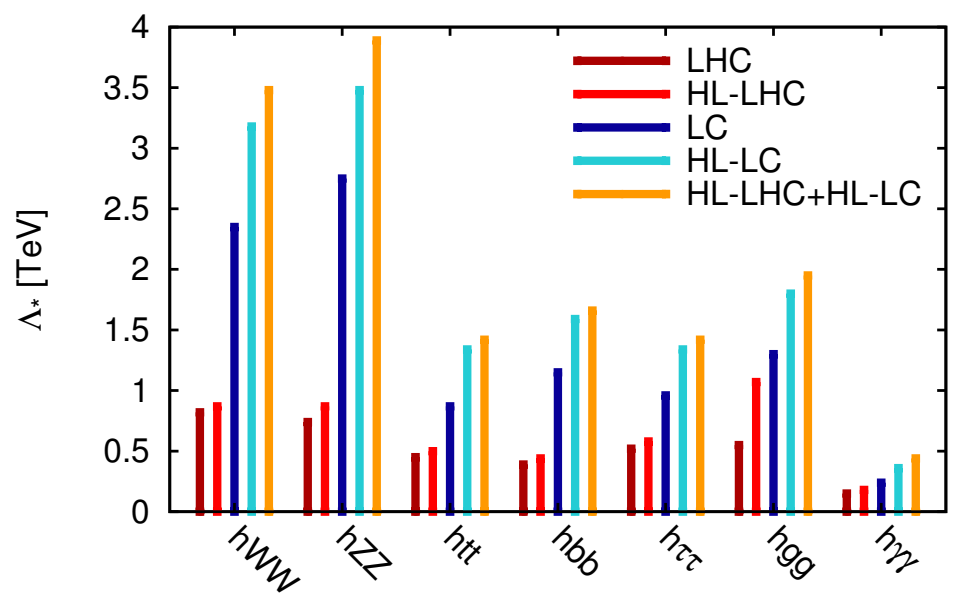

FIG. 2: Effective new physics scales $\Lambda_{*}$ extracted from the Higgs coupling measurements collected in TableI The values for the loop-induced couplings to gluons and photons contain only the contribution of the contact terms, as the effects of the loop terms are already disentangled at the level of the input values $\Delta$. (The ordering of the columns from left to right corresponds to the legend from up to down.) 
An elegant formulation inspired by the AdS/CFT correspondence [72] allows us to define a strongly interacting Higgs sector in four space-time dimensions via a Randall-Sundrum setup [73] i.e. a slice of five dimensional anti-de Sitter space bounded by two 3-branes 29 31. The 5-dimensional picture can be used to investigate the dynamics of the theory, which is not straightforwardly accessible in the strongly coupled 4-dimensional picture [32. By using the standard AdS/CFT dictionary (see e.g. Ref. [74]) we can construct a low energy effective theory that meets the phenomenologically observed symmetry requirements, which can now be understood in a strongly interacting [75] large- $N$ conformal field theory (CFT) context. The IR brane-localized modes in the $5 \mathrm{~d}$ picture correspond to additional composite states that are indispensable for unitarity conservation in the model, but can be neglected in a low-energy EFT approach. The relevant small parameter in the light Higgs effective theory is $v / f$, where in the most strongly interacting setup the scale of the additional states is $4 \pi f$.

Another way to model a strongly interacting Higgs sector with light Goldstone modes is by applying QCDinspired chiral perturbation theory. In contrast to our discussion in Section 2.1 such a chiral Lagrangian is usually not written based on the linear representation of the Higgs and Goldstone fields and hence does not include an $S U(2)_{L}$ doublet $\phi$. The Higgs field then appears as a singlet in the combination $h / v$, which gives more freedom to define operators and does not allow for a one-to-one correspondence of the power counting of the linear Higgs operators and the non-linear chiral Lagrangian. The non-linear and linear sets of interaction operators are mutually equivalent concerning all possible Lorentz and $U(1)_{e m}$ invariant couplings. If instead one only considers the leading components in each set, there is only partial correspondence between the leading operators [46, 52, 76].

As a specific example, where a one-to-one correspondence of leading operators still holds, we show an ansatz for the higher-dimensional Lagrangian based on the extra-dimensional strongly interacting theory described above. Its leading terms have the same form as they would have in an effective field theory based on $\phi^{\dagger} \phi / f^{2}$ in a linear representation. In the experimentally least vulnerable case where the mass scale of the additional Kaluza-Klein states is given by $m_{\rho} \sim 4 \pi f$ and we omit operators violating custodial symmetry, the strongly interacting Higgs Lagrangian includes the leading dimension-6 operators [59]

$$
\mathcal{L}_{\mathrm{SILH}} \supset \frac{c_{h}}{2 f^{2}}\left[\partial^{\mu}\left(h^{\dagger} h\right)\right]^{2}-\frac{c_{6} \lambda}{f^{2}}\left(h^{\dagger} h\right)^{3}+\sum_{f}\left(\frac{c_{y} y_{f}}{f^{2}} h^{\dagger} h \bar{f}_{L} h f_{R}+\text { h.c. }\right)+\mathcal{O}\left(\frac{1}{(4 \pi f)^{2}}\right)+\mathcal{O}\left(\frac{1}{\left(16 \pi^{2} f\right)^{2}}\right)
$$

where $c_{h}, c_{6}, c_{y}$ are numbers of order unity and $\lambda, y_{f}$ denote the Higgs self-coupling and the Yukawa couplings, respectively.

Even without using any equations of motions the structure of the leading corrections to the Standard Model Higgs couplings is relatively simple. Deviations in the couplings to massive gauge bosons are protected by custodial symmetry. The leading deviations affect the Higgs self-coupling and the Yukawa couplings, corresponding to the reduced operator basis $\left\{\mathcal{O}_{\phi 2}, \mathcal{O}_{\phi 3}, \mathcal{O}_{L R}\right\}$ as compared to the basis defined in Eq.2.6. In addition, the first term in Eq.2.14 generates a Higgs wave function renormalization, which leads to a universal correction of all Higgs couplings as $1+\Delta=\left(1-c_{h} v^{2} / f^{2}\right)^{1 / 2}$. The phenomenologically relevant gauge boson and fermion couplings are modified by the two parameters $\xi$ and $\xi c_{y} / c_{h}$ [77, where

$$
\xi=c_{h}\left(\frac{v}{f}\right)^{2}
$$

is related to the Goldstone scale $f$ relative to the standard Higgs vacuum expectation value $v$. Compared to the weakly interacting models discussed before, this two-parameter setup corresponds to the simplest $2 \mathrm{HDM}$ scenarios [19].

The ratio $c_{y} / c_{h}$ can be predicted in the context of holographic Higgs Models, in which strongly coupled theories in four dimensions are identified with weakly coupled theories in five dimensions. In theories in which the global symmetry $\mathrm{SO}(5)$ is broken to $\mathrm{SO}(4)$, the Standard Model fermions may be assigned either to spinorial or fundamental $\mathrm{SO}(5)$ representations, changing the Higgs couplings either universally $\left(c_{y} / c_{h}=0\right)$ or 


\begin{tabular}{|l||c|c||c|c||c|}
\hline$\xi$ & LHC & HL-LHC & LC & HL-LC & HL-LHC+HL-LC \\
\hline universal & 0.076 & 0.051 & 0.008 & 0.0052 & 0.0052 \\
non-universal & 0.068 & 0.015 & 0.0023 & 0.0019 & 0.0019 \\
\hline \hline$f[\mathrm{TeV}]$ & & & & & \\
\hline universal & 0.89 & 1.09 & 2.82 & 3.41 & 3.41 \\
non-universal & 0.94 & 1.98 & 5.13 & 5.65 & 5.65 \\
\hline
\end{tabular}

TABLE IV: Estimates of the parameter $\xi=(v / f)^{2}$ and the Goldstone scale $f$ for various experimental set-ups and two different fermion embeddings (universal, non-universal).

separately $\left(c_{y} / c_{h} \neq 0\right)$ for Standard Model vectors and fermions. The spinorial case where all Higgs couplings are suppressed universally by a factor $(1-\xi)^{1 / 2}[30$ is covered by the analysis of portal models.

In a closely related scenario [31] universality is broken to the extent that the Higgs coupling of vector particles is reduced by the standard coefficient, but the coupling of fermions by a different coefficient,

$$
1+\Delta_{V}=\sqrt{1-\xi} \approx 1-\frac{\xi}{2} \quad 1+\Delta_{f}=\frac{1-2 \xi}{\sqrt{1-\xi}} \approx 1-3 \frac{\xi}{2} .
$$

for $\xi \ll 1$. Based on the estimates of potential deviations from SM Higgs couplings, bounds on the parameter $\xi$ and the ensuing scale $f$ are presented in Table IV.

The typical bounds on $\xi$ range from $7.6 \%$ at the LHC up to 2 permille for the non-universal scenario at an upgraded linear collider. The expectations in the non-universal scenario are stronger than those in the universal scenario, apparent from the factor 3 in the expanded version of $\Delta_{f}$ in Eq. 2.16 compared with $\Delta_{V}$ in both scenarios. In terms of the Goldstone scale $f$ this corresponds to scales between just under a $\mathrm{TeV}$ to more than $5 \mathrm{TeV}$. These numbers exceed the limits from electroweak precision data, which yield $f \gtrsim 750 \mathrm{GeV}[56$. However, no meaningful limit can be obtained from LHC Higgs data if we relax the assumption that there are no non-SM Higgs decay modes [61, 77.

\section{MIXING EFFECTS}

Mixing phenomena are a general consequence of multi-field structures in the scalar sector. Assuming that one of the states is essentially identical with the SM Higgs state, mixing nevertheless affects masses and couplings, inducing potentially small deviations from the SM values for large scales of the new physics sector. These mixing effects can in principle be quite complex. We will discuss in detail three interesting examples which illustrate the basic features.

\subsection{Higgs portal}

The large dark component of matter in the Universe strongly suggests a dark sector with potentially complex structure [78. This sector may interact with the Standard-Model sector through the Higgs portal [24 27], the two sectors coupled by a renormalizable quartic interaction,

$$
\mathcal{L}_{p}=-\eta\left|\phi_{s}\right|^{2}\left|\phi_{d}\right|^{2}
$$

between the SM Higgs field $\phi_{s}$ and a corresponding dark Higgs field $\phi_{d}$. The individual interactions conform with the standard quartic interactions of spontaneous symmetry breaking with strengths $\lambda_{s}$ and $\lambda_{d}$,

$$
\mathcal{L}_{4}=-\frac{\lambda_{s}}{2}\left|\phi_{s}\right|^{4}-\frac{\lambda_{d}}{2}\left|\phi_{d}\right|^{4} .
$$


Before symmetry breaking the theory is $S U(3) \times S U(2) \times U(1)$ gauge-invariant. After symmetry breaking in the SM and hidden sectors the system is described by the mass matrix [26]

$$
\mathcal{M}^{2}=\left(\begin{array}{cc}
\lambda_{s} v_{s}^{2} & \eta v_{s} v_{d} \\
\eta v_{s} v_{d} & \lambda_{d} v_{d}^{2}
\end{array}\right)
$$

where $v_{s} \equiv v^{\mathrm{SM}}$ and $v_{d}$ are the vacuum expectation values of the Higgs fields of the coupled system, the coupling mediated by the mixed term $\eta$.

The mass spectrum of the two Higgs bosons,

$$
\begin{aligned}
& m_{s_{1}}^{2} \simeq \lambda_{s} v_{s}^{2}-\eta^{2} v_{s}^{2} / \lambda_{d} \\
& m_{d_{1}}^{2} \simeq \lambda_{d} v_{d}^{2}+\eta^{2} v_{s}^{2} / \lambda_{d}
\end{aligned}
$$

splits characteristically into a light SM-type state and a new heavy state. The initial masses in the two sectors are pulled apart by the mutual interaction, inducing a mass splitting of the order $v_{s}$ times the interaction strength $\eta$. Thus, the mixing effect on the mass spectrum is determined by the light SM scale and not by the heavy scale, and a sufficiently small mixing parameter $\eta$ allows the light system to approach the structure of the Standard Model.

The two mass eigen-fields $s_{1}, d_{1}$ are rotated out of the current fields $s, d$ by

$$
\begin{aligned}
s_{1} & =+\cos \chi s+\sin \chi d \\
d_{1} & =-\sin \chi s+\cos \chi d,
\end{aligned}
$$

with the mixing angle $\chi$ given by

$$
\tan (2 \chi) \simeq-\frac{2 \eta v_{s}}{\lambda_{d} v_{d}}
$$

This size of the phenomenological mixing angle is determined by $\eta$ and, in contrast to the mass spectrum, by the ratio $v_{s} / v_{d}$ of the SM scale over the high scale, as naively expected. The mixing affects all the couplings of the SM-like Higgs boson universally,

$$
g_{s_{1}}=\cos \chi g_{h}^{\mathrm{SM}},
$$

which is certainly the easiest way to quantify large deviations from the Standard Model in existing experimental data. The present bound on $\cos ^{2} \chi$ is shown in the left panel of Fig. 3 . Despite the fact that the mass scale $v_{d}$ is much larger than $v_{s}$, there could still be light particles in the dark sector, just like all SM fermions but the top quark have a mass much smaller than $v$. This opens up the possibility for invisible decays of the SM-like state $s_{1}$. Therefore, we combine it with the estimate of the partial width for invisible Higgs decay channels. The improvements foreseen from LHC, HL-LHC, LC and HL-LC in the coming years and later in the future are displayed in Fig. 3 (right), reinterpreting the results given in Table I. It is apparent that a fine-grain picture of the Higgs boson can be drawn by analyzing the couplings.

Denoting $\delta_{\chi}=\sqrt{1+\tan ^{2}(2 \chi)}-1$, which reduces for small mixing to $\delta_{\chi} \simeq 2 \chi^{2}$, the individual Higgs vacuum expectation values are shifted by

$$
\begin{aligned}
& \lambda_{s} v_{s}^{2}=m_{s_{1}}^{2}+\frac{1}{2} m_{d_{1}}^{2} \delta_{\chi} \\
& \lambda_{d} v_{d}^{2}=m_{d_{1}}^{2}-\frac{1}{2} m_{d_{1}}^{2} \delta_{\chi}
\end{aligned}
$$

for $m_{d_{1}} \gg m_{s_{1}}$. Evidently, the measurement of the light Higgs mass $m_{s_{1}}$ and the mixing parameter $\delta_{\chi}$ gives rise to an upper limit on the heavy Higgs mass $m_{d_{1}}$

$$
m_{d_{1}}^{2} \leq \frac{2 m_{s_{1}}^{2}}{\delta_{\chi}}
$$



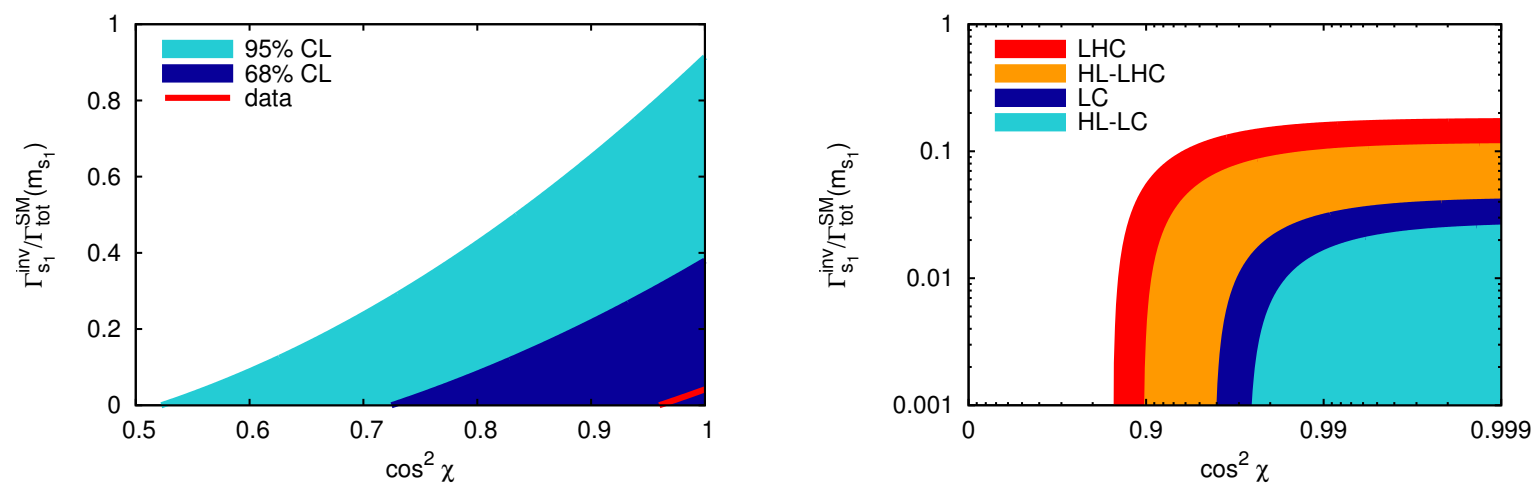

FIG. 3: Best-fit contours for the mixing parameter $\cos ^{2} \chi$ and the hidden decay width $\Gamma^{\text {inv }}$ in the Higgs portal model. Left: Shown is the relation originating from the best-fit point of the Higgs couplings and the $68 \%$ and $95 \%$ C.L. error bars, using the result of Ref. [19], which includes LHC data up to around Moriond/Aspen 2013. Right: Expected improvements at the $95 \%$ C.L. from future running of LHC, HL-LHC, LC and HL-LC.
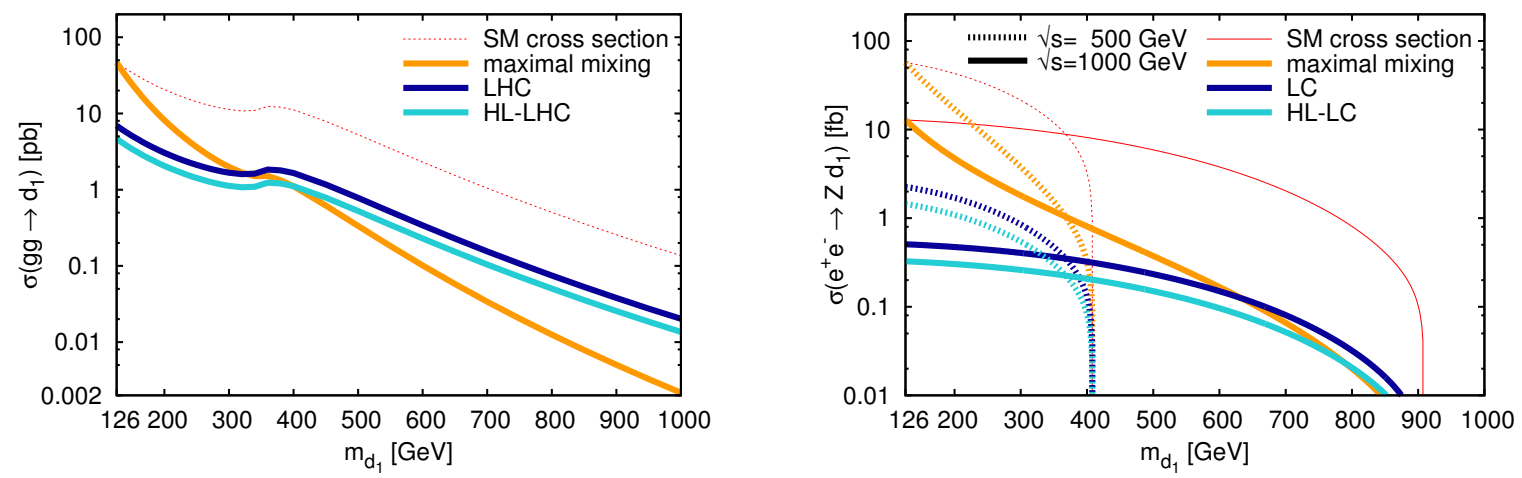

FIG. 4: Production cross sections of the heavy Higgs boson in portal models, as a function of the Higgs mass (left) at the LHC; (right) at the LC. The yellow curve shows the $d_{1}$ production cross section for maximal allowed mixing according to Eq. 3.9, while the blue curves correspond to the maximal allowed mixing if the $s_{1}$ couplings agree with the Standard Model within $95 \%$ C.L. after the standard and high-luminosity run of the respective collider, see Eq.(3.7).

As naturally expected in quantum mechanics, the masses approach each other for large mixing while the gap spreads for small mixing.

The interplay between heavy Higgs masses and mixings in observing this state either at LHC or LC is demonstrated in Fig. 4 As a function of the Higgs mass, the mixing parameters are chosen according to three different scenarios. These are the maximal allowed mixings given by Eq. 3.9p and assuming that the $s_{1}$ couplings agree with the Standard Model at the 95\% C.L. after both the standard and high-luminosity run of the respective collider. For the LHC we show the gluon fusion Higgs production cross sections including NNLO and NNLL QCD corrections [79], while for the LC the Higgs-strahlung process $e^{+} e^{-} \rightarrow Z+h$ is presented with cross sections of 67.1 and $13.4 \mathrm{fb}$ for a SM Higgs boson with a mass of $126 \mathrm{GeV}$ at LC facilities of energies 500 $\mathrm{GeV}$ and $1 \mathrm{TeV}[14$, respectively. 


\begin{tabular}{|c|cccc|}
\hline$\frac{\Gamma_{2 \mathrm{HDM}}\left[h^{0} \rightarrow X\right]}{\Gamma_{\mathrm{SM}}[h \rightarrow X]}$ & type I & type II & lepton-spec. & flipped \\
\hline$V V^{*}$ & $\sin ^{2}(\beta-\alpha)$ & $\sin ^{2}(\beta-\alpha)$ & $\sin ^{2}(\beta-\alpha)$ & $\sin ^{2}(\beta-\alpha)$ \\
$\bar{u} u$ & $\frac{\cos ^{2} \alpha}{\sin ^{2} \beta}$ & $\frac{\cos ^{2} \alpha}{\sin ^{2} \beta}$ & $\frac{\cos ^{2} \alpha}{\sin ^{2} \beta}$ & $\frac{\cos ^{2} \alpha}{\sin ^{2} \beta}$ \\
$\bar{d} d$ & $\frac{\cos ^{2} \alpha}{\sin ^{2} \beta}$ & $\frac{\sin ^{2} \alpha}{\cos ^{2} \beta}$ & $\frac{\cos ^{2} \alpha}{\sin ^{2} \beta}$ & $\frac{\sin ^{2} \alpha}{\cos ^{2} \beta}$ \\
$\ell^{+} \ell^{-}$ & $\frac{\cos ^{2} \alpha}{\sin ^{2} \beta}$ & $\frac{\sin ^{2} \alpha}{\cos ^{2} \beta}$ & $\frac{\sin ^{2} \alpha}{\cos ^{2} \beta}$ & $\frac{\cos ^{2} \alpha}{\sin ^{2} \beta}$ \\
\hline
\end{tabular}

TABLE V: Partial widths of the light Higgs boson $h^{0}$ in different realizations of the 2HDM, relative to the SM widths at leading order.

\subsection{Two Higgs doublets and the MSSM}

In a general Two-Higgs-Doublet model (2HDM), the physical states are mixtures of the components of the two doublets $\phi_{1}$ and $\phi_{2}[22,23]$. The scalar potential can be written as

$$
\begin{aligned}
V= & m_{11}\left|\phi_{1}\right|^{2}+m_{22}^{2}\left|\phi_{2}\right|^{2}-m_{12}^{2}\left(\phi_{1}^{\dagger} \phi_{2}+\text { h.c }\right)+\lambda_{1}\left|\phi_{1}\right|^{4}+\lambda_{2}\left|\phi_{2}\right|^{4} \\
& +\lambda_{3}\left|\phi_{1}\right|^{2}\left|\phi_{2}\right|^{2}+\lambda_{4}\left|\phi_{1}^{\dagger} \phi_{2}\right|^{2}+\frac{1}{2} \lambda_{5}\left[\left(\phi_{1}^{\dagger} \phi_{2}\right)^{2}+\text { h.c }\right] .
\end{aligned}
$$

The Higgs-fermion couplings depend on the specific type of the 2HDM. To ensure natural suppression of flavorchanging neutral currents, one usually demands that one type of fermions couples only to one Higgs doublet. This pattern can be imposed by a global $\mathbb{Z}_{2}$ discrete symmetry, under which $\phi_{1,2} \rightarrow \mp \phi_{1,2}$, and which has been assumed in the potential Eq. (3.10). So all terms in Eq. 3.10) include an even power of each of the Higgs fields $\phi_{1}$ and $\phi_{2}$. Both Higgs fields acquire vacuum expectations values, $v_{1}$ and $v_{2}$, with $v_{1}^{2}+v_{2}^{2}=v^{2}$ and $\tan \beta=v_{2} / v_{1}$. Depending on the $\mathbb{Z}_{2}$ charge assignments, the following four cases of coupling the Higgs doublets to fermions are possible [80:

- type I: all fermions couple only to $\phi_{2}$;

- type II: up-/down-type fermions couple to $\phi_{2} / \phi_{1}$, respectively;

- lepton-specific: quarks couple to $\phi_{2}$ and charged leptons couple to $\phi_{1}$;

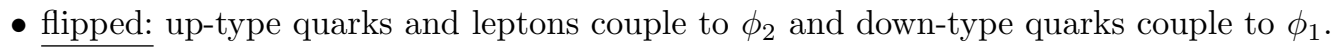

After electroweak symmetry breaking the Higgs sector consists of three neutral Higgs bosons, two CP-even ones $h^{0}, H^{0}$ and a CP-odd one $A^{0}$, as well as of two charged Higgs bosons $H^{ \pm}$. Leaving aside modifications of the loop decays from the individual loop particle contributions and ignoring Higgs-to-Higgs decays, which alter the branching ratios, the partial widths of the light scalar $h^{0}$ are modified relative to the Standard Model through mixing effects of the two Higgs doublets. They can be expressed in terms of $\beta=\arctan v_{2} / v_{1}$ and $\alpha$, the mixing angle between the two CP-even Higgs bosons $h_{0}$ and $H_{0}$, see Table $\mathrm{V}$.

If the heavy Higgs bosons $\left(H^{0}, A^{0}\right.$, and $\left.H^{ \pm}\right)$have masses much larger than $v$, one enters a regime where the physics of the light Higgs $h^{0}$ can be described by an effective theory [19, 23. In this case, commonly known as the decoupling limit, the heavy Higgs masses are approximately given by

$$
m_{A^{0}}^{2}, m_{H^{0}}^{2}, m_{H^{ \pm}}^{2}=\frac{2 m_{12}^{2}}{\sin (2 \beta)}+\mathcal{O}\left(v^{2}\right),
$$

while the properties of the lightest CP-even Higgs boson $h^{0}$ are close to the Standard Model. The leading modification of the partial widths of $h^{0}$ in relation to the Standard Model can be expressed as an expansion in the parameter

$$
\xi=\frac{v^{2}}{2 m_{A^{0}}^{2}} \sin ^{2}(2 \beta)\left[\lambda_{1}-\lambda_{2}+\left(\lambda_{1}+\lambda_{2}-\lambda_{3}-\lambda_{4}-\lambda_{5}\right) \cos 2 \beta\right]
$$


For the factors in Table $\nabla$ one thus finds

$$
\sin ^{2}(\beta-\alpha) \approx 1-\xi^{2}, \quad \frac{\cos ^{2} \alpha}{\sin ^{2} \beta} \approx 1+2 \xi \cot \beta, \quad \frac{\sin ^{2} \alpha}{\cos ^{2} \beta} \approx 1-2 \xi \tan \beta .
$$

Numerically, for $\lambda_{i} \sim \mathcal{O}(1)$ and $\tan \beta \approx 1$, the parameter $\xi \approx 0.03 /\left(m_{A^{0}} / \mathrm{TeV}\right)^{2}$, so one expects corrections of tens of percent for moderate values of $m_{A^{0}}$ and $\tan \beta$. The shape of the decoupling limit in the different model setups can be seen in Fig. 5. The preference for type-I models in a comparably wide parameter range is that it separates Higgs couplings to gauge bosons and fermions and makes it easy to accommodate the slightly enhanced $H \rightarrow \tau \tau$ rate. Many dedicated 2HDM analyses based on the Higgs couplings measured at the LHC are available for the different model setups [82]. Given the generic size of experimental error bars and the fact that after the recent experimental updates all channels are in broad agreement with the Standard Model predictions, none of them shows a clear sign for such mixing effects.

Minimal Supersymmetric Models (MSSM) form a subgroup of the general 2HDM type-II. The quartic couplings of the $2 \mathrm{HDM}$ scalar potential are restricted to special values given by the $S U(2)_{L}$ and $U(1)$ gauge couplings $g$ and $g^{\prime}$,

$$
\lambda_{1}=\lambda_{2}=-\frac{1}{2} \lambda_{3}=\frac{1}{8}\left(g^{2}+g^{\prime 2}\right), \quad \lambda_{4}=-\lambda_{5}=-g^{2} .
$$

Because just like in the Standard Model the Higgs masses are determined by the quartic couplings, this structure predicts the maximum mass of the lightest Higgs scalar [83. In the decoupling limit, i.e. the limit of heavy Higgs bosons $H^{0}, A^{0}$ and $H^{ \pm}$, the partial widths of this SM-like light state scale as

$$
\begin{aligned}
& \frac{\Gamma_{\mathrm{SUSY}}\left[h^{0} \rightarrow V V^{*}\right]}{\Gamma_{\mathrm{SM}}\left[h \rightarrow V V^{*}\right]} \approx 1-\frac{m_{Z}^{4} \sin ^{2} 2 \beta}{m_{A^{0}}^{4}}\left(\cos 2 \beta+R_{t}\right)^{2}, \\
& \frac{\Gamma_{\mathrm{SUSY}}\left[h^{0} \rightarrow u u\right]}{\Gamma_{\mathrm{SM}}[h \rightarrow u u]} \approx 1+\frac{4 m_{Z}^{2} \cos ^{2} \beta}{m_{A^{0}}^{2}}\left(\cos 2 \beta+R_{t}\right), \\
& \frac{\Gamma_{\mathrm{SUSY}}\left[h^{0} \rightarrow d d\right]}{\Gamma_{\mathrm{SM}}[h \rightarrow d d]} \approx 1-\frac{4 m_{Z}^{2} \sin ^{2} \beta}{m_{A^{0}}^{2}}\left(\cos 2 \beta+R_{t}\right),
\end{aligned}
$$

where $R_{t}$ captures the SUSY radiative corrections, which are dominated by the top/stop loop contributions:

$$
\begin{aligned}
R_{t} \approx \frac{3\left(g^{2}+g^{\prime 2}\right)}{16 \pi^{2} \sin ^{2} \beta} \frac{m_{t}^{4}}{m_{Z}^{4}}[ & \log \frac{m_{\tilde{t}_{1}} m_{\tilde{t}_{2}}}{m_{t}^{2}}+\left(A_{t}-\mu \cot 2 \beta\right) \frac{A_{t}-\mu \cot \beta}{m_{\tilde{t}_{1}}^{2}-m_{\tilde{t}_{2}}^{2}} \log \frac{m_{\tilde{t}_{1}}^{2}}{m_{\tilde{t}_{2}}^{2}} \\
& \left.+\left(A_{t}^{2}-\mu^{2}-2 A_{t} \mu \cot 2 \beta\right)\left(\frac{A_{t}-\mu \cot \beta}{m_{\tilde{t}_{1}}^{2}-m_{\tilde{t}_{2}}^{2}}\right)^{2}\left(1-\frac{m_{\tilde{t}_{1}}^{2}+m_{\tilde{t}_{2}}^{2}}{m_{\tilde{t}_{1}}^{2}-m_{\tilde{t}_{2}}^{2}} \log \frac{m_{\tilde{t}_{1}}}{m_{\tilde{t}_{2}}}\right)\right],
\end{aligned}
$$

where $\mu$ denotes the higgsino mass parameter, $m_{\tilde{t}_{1,2}}$ the stop masses and $A_{t}$ the soft SUSY breaking trilinear coupling of the stop sector. To achieve compatibility with the relatively 'large' Higgs boson mass of $m_{h} \simeq$
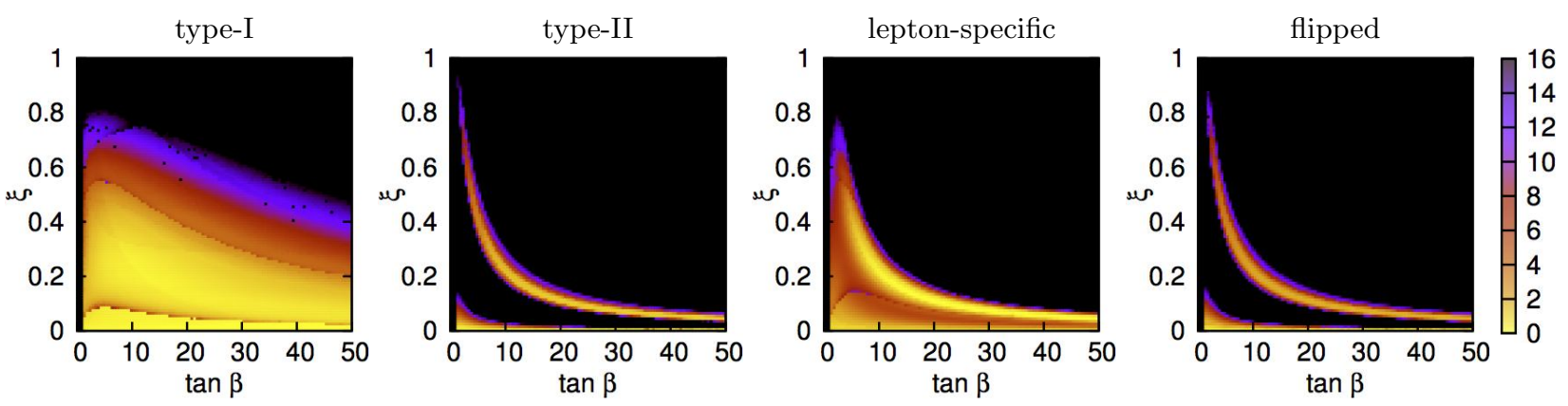

FIG. 5: Allowed ranges for the modification parameter $\xi$ in a 2HDM in the decoupling limit, based on data from Ref. 81. The plots show the correlated relative $\log$-likelihood $-2 \Delta(\log \mathcal{L})$ as a function of $\tan \beta$, from Ref. [19]. 


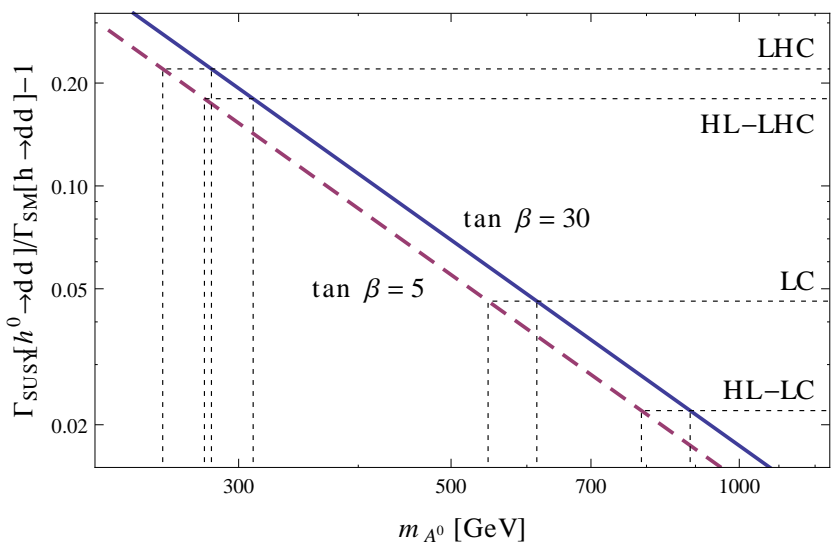

FIG. 6: Dependence of the Higgs tree-level decay width into down-type fermions on $m_{A^{0}}$ within the MSSM, for two examples of $\tan \beta=5$ and 30 , as well as $m_{\tilde{t}_{1}} m_{\tilde{t}_{2}}=1 \mathrm{TeV}^{2}$ and $A_{t}-\mu \cot \beta \ll m_{\tilde{t}_{i}}$. Also shown are the expected limits from the LHC, HL-LHC, LC, HL-LC.

$126 \mathrm{GeV}$ in the MSSM, rather high scales $m_{\tilde{t}_{1,2}} \sim \mathcal{O}(1 \mathrm{TeV})$ for the stop masses help, at the same time enhancing the corrections in $R_{t}$.

Due to the small number of free parameters in the tree-level Higgs sector of the MSSM, as compared to the $2 \mathrm{HDM}$, one can extract significant information on the supersymmetric parameter space from LHC measurements. A strong constraint comes from the light Higgs mass, but additional information is obtained from the measured Higgs couplings in such a parameter study [84 [86]. In Fig. 66 we show how the CP-odd mass $m_{A^{0}}$ can be estimated from measurements of the light Higgs couplings. This is illustrated for two representative values $\tan \beta=5$ and 30. For large values of $\tan \beta$ we need to keep in mind that vertex loop corrections can become equally important as the tree-level mixing effects, see Section 4.2 .

\subsection{Next-to-Minimal Supersymmetric Standard Model (NMSSM)}

In the NMSSM [87 89] an additional singlet superfield $S$ is added to the MSSM spectrum. The $\mu$ parameter is generated dynamically through the coupling of $S$ to the two Higgs doublet fields, $\lambda S H_{u} H_{d}$. In order to avoid a massless axion, the Peccei-Quinn symmetry [90] is broken by the introduction of a cubic coupling of the singlet field $S, \kappa S^{3} / 3$, in the scale-invariant superpotential. Furthermore, the MSSM soft SUSY breaking Lagrangian is extended by a soft SUSY breaking mass term and trilinear soft SUSY breaking interactions for the singlet field,

$$
-\mathcal{L}_{S, \text { soft }}=m_{S}^{2}|S|^{2}+\lambda A_{\lambda} H_{u} H_{d} S+\frac{1}{3} \kappa A_{\kappa} S^{3} .
$$

After electroweak symmetry breaking the Higgs sector consists of seven Higgs bosons, three CP-even states $H_{i}$ $(i=1,2,3)$, two CP-odd bosons $A_{1}$ and $A_{2}$, and two charged Higgs bosons $H^{ \pm}$. The CP-even/odd states are ordered by ascending mass. Depending on the choice of parameters, either the lightest or the next-to-lightest Higgs boson can be the SM-like Higgs $h$. Its upper mass bound is given by

$$
m_{h}^{2} \approx M_{Z}^{2} \cos ^{2} 2 \beta+\frac{\lambda^{2} v^{2}}{2} \sin ^{2} 2 \beta+\Delta m_{h}^{2},
$$

where $v \approx 246 \mathrm{GeV}$. The tree-level contribution is maximized for small values of $\tan \beta$, and due to the additional term proportional to $\lambda$ a radiative correction $\Delta m_{h}^{2}$ of only $\sim(75 \mathrm{GeV})^{2}$ is required to achieve $m_{h} \approx 126 \mathrm{GeV}$, for $\lambda=0.6$ and $\tan \beta=2$. The CP-even/CP-odd Higgs mass eigenstates are admixtures of the singlet components $h_{s} / a_{s}$ and the doublet components $h_{u}, h_{d} / a_{u}, a_{d}$, leading to suppressed couplings to the SM particles due to the singlet component. 

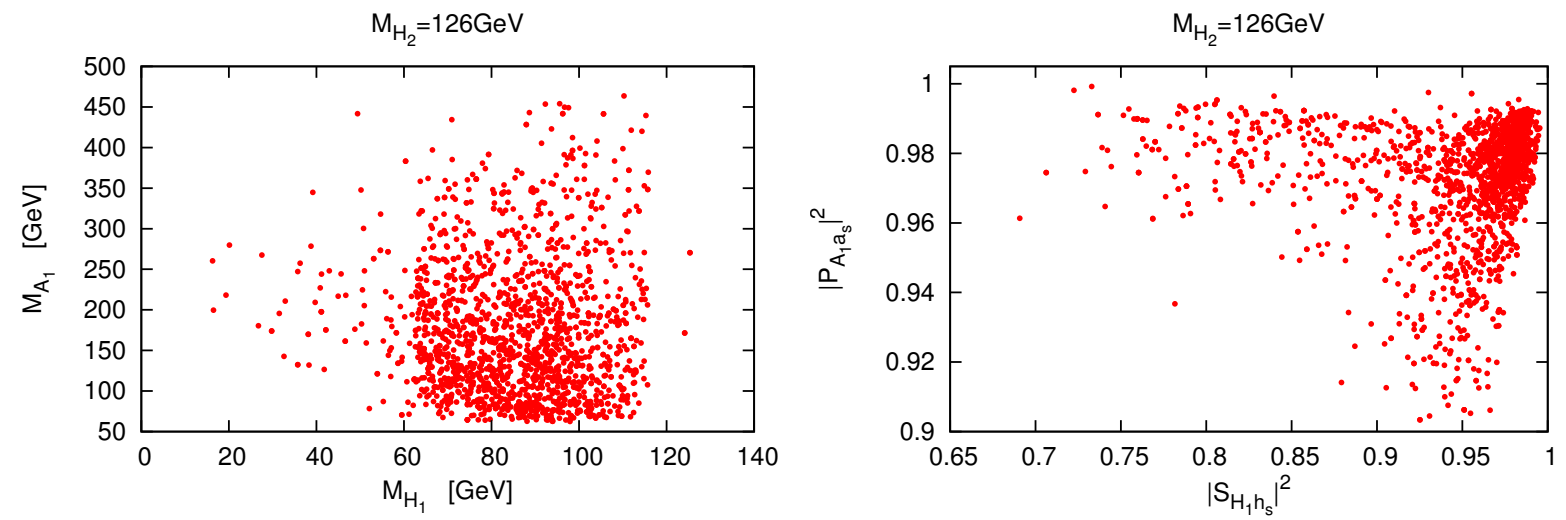

FIG. 7: Left: Mass values of $A_{1}$ and $H_{1}$ in GeV. Right: The singlet admixtures $\left|P_{A_{1} a_{s}}\right|^{2}$ and $\left|S_{H_{1} h_{s}}\right|^{2}$ of the lightest pseudoscalar $A_{1}$ and of the scalar $H_{1}$.

In this section we will first summarize the evidence that the experimental observation of the 126-GeV Higgs state and the measurement of its couplings are compatible with the NMSSM. And second, that sum rules for precisely measured couplings can be exploited to prove that observing three neutral MSSM-type Higgs bosons does not close the system but may point to [two] more heavy neutral particles as predicted in the NMSSM.

The present LHC Higgs search results [2] can be accommodated in the NMSSM Higgs sector [91, 92]. With one of the CP-even Higgs bosons being SM-like, the LEP constraints can be avoided for the light CP-even and CP-odd Higgs states because of sizeable singlet admixtures. The heavy MSSM-like Higgs states could have avoided discovery due to too small cross sections because of missing phase space for their production or singlet admixtures in their couplings to the SM particles. ${ }^{\dagger}$ Figures 7 show the results of a scan over a subspace of the NMSSM parameter space:

$$
\begin{array}{lrl}
1 \leq \tan \beta \leq 30, & 100 \mathrm{GeV} \leq \mu \leq 500 \mathrm{GeV}, & 100 \mathrm{GeV} \leq M_{1} \leq 1 \mathrm{TeV} \\
0.5 \leq \lambda \leq 0.8, & -500 \mathrm{GeV} \leq A_{\lambda} \leq 800 \mathrm{GeV}, & 200 \mathrm{GeV} \leq M_{2} \leq 1 \mathrm{TeV} \\
0 \leq \kappa \leq 0.8, & -500 \mathrm{GeV} \leq A_{\kappa} \leq 200 \mathrm{GeV}, & 1.1 \mathrm{TeV} \leq M_{3} \leq 2 \mathrm{TeV}
\end{array}
$$

and

$$
\begin{aligned}
& -2 \mathrm{TeV} \leq A_{U}, A_{D}, A_{E} \leq 2 \mathrm{TeV} \quad M_{\tilde{\mu}_{R}, \tilde{e}_{R}}=M_{\tilde{L}_{1,2}}=M_{\tilde{Q}_{1,2}}=M_{\tilde{c}_{R}, \tilde{u}_{R}}=M_{\tilde{s}_{R}, \tilde{d}_{R}}=2.5 \mathrm{TeV} \\
& 500 \mathrm{GeV} \leq M_{\tilde{t}_{R}}=M_{\tilde{Q}_{3}} \leq 1.5 \mathrm{TeV}, \quad 500 \mathrm{GeV} \leq M_{\tilde{\tau}_{R}}=M_{\tilde{L}_{3}} \leq 1 \mathrm{TeV}, \quad M_{\tilde{b}_{R}}=1 \mathrm{TeV}
\end{aligned}
$$

Here $M_{i}(i=1,2,3)$ denote the soft SUSY breaking gaugino masses, $M_{\tilde{x}_{R}}$ and $M_{\tilde{X}_{j}}(j=1,2,3)$ the righthanded and left-handed soft SUSY breaking sfermion masses and $A_{U, D, E}$ the trilinear soft SUSY breaking couplings of the up- and down-type quarks and the charged leptons. The thus generated squark and gluino masses have not been excluded yet by the LHC searches. The scan leads to scenarios where either the lightest Higgs boson $H_{1}$ or the second lightest $H_{2}$ has a mass of $126 \mathrm{GeV}$ and signal rates that are compatible within $1 \sigma$ with the rates reported by ATLAS and CMS. In the following, we present results for the latter case. The scenarios with a SM-like $H_{1}$ lead to similar conclusions. Figure 7 (left) shows the mass values of the lightest scalar and pseudoscalar Higgs bosons $A_{1}$ and $H_{1}$. The former ranges between $\sim 62$ and $\sim 480 \mathrm{GeV}$, while $M_{H_{1}} \gtrsim 18 \mathrm{GeV}$ with the upper bound given by the $H_{2}$ mass of $126 \mathrm{GeV}$. In the case where $H_{1}$ and $H_{2}$ are almost degenerate in mass, the Higgs signal observed at the LHC is built up by two Higgs bosons. The LEP exclusion limits are avoided due to $H_{1}$ and $A_{1}$ being rather singlet-like, which leads to suppressed couplings

\footnotetext{
† A further reduction of branching ratios into SM particle final states, and hence discovery signatures, can be due to possible Higgs-to-Higgs decays.
} 

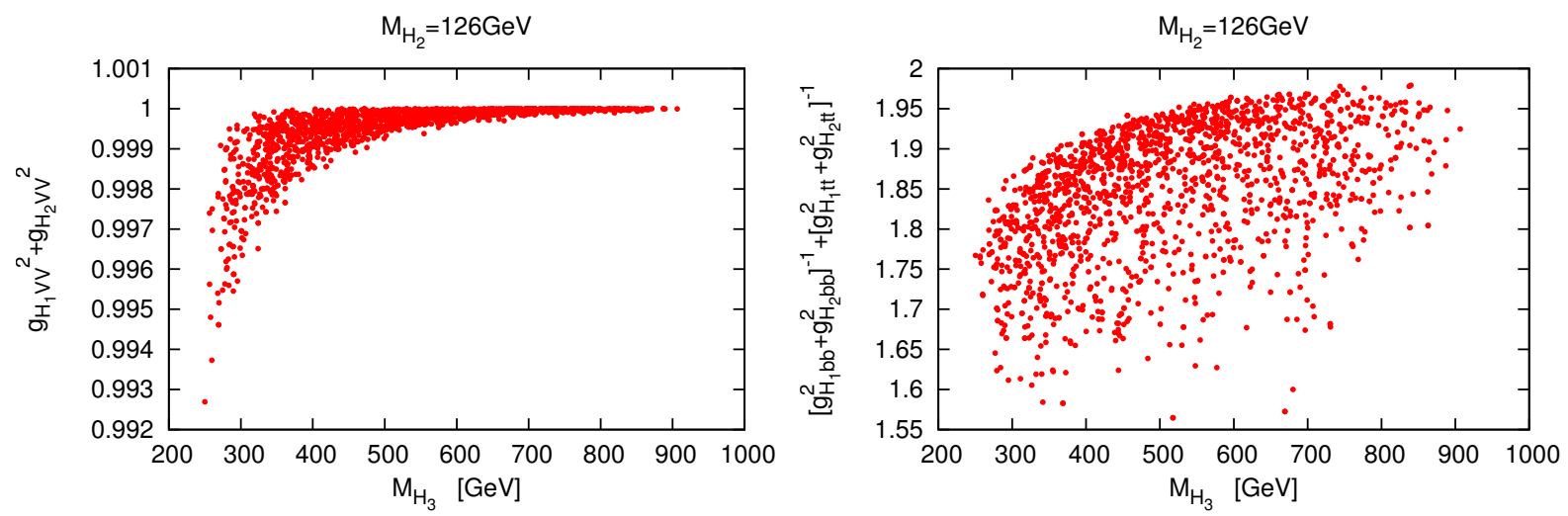

FIG. 8: Left: Sum of the $H_{1}$ and $H_{2}$ (=SM-like) gauge couplings squared. Right: Sum of the inverse $H_{1}$ and $H_{2}$ Yukawa couplings squared. Both are shown as a function of $M_{H_{3}}$.

and small signal rates. The singlet components for $A_{1}$ and $H_{1}$ are shown in Fig. 7 (right). They are quantified by the corresponding matrix elements squared, $\left|P_{A_{1} a_{s}}\right|^{2}$ and $\left|S_{H_{1} h_{s}}\right|^{2}$, of the mixing matrices $P$ and $S$, which rotate the $\mathrm{CP}$-odd and $\mathrm{CP}$-even Higgs interaction states to the mass eigenstates. As anticipated, $A_{1}$ and $H_{1}$ are rather singlet-like. The masses of the heavy Higgs bosons $A_{2}$ and $H_{3}$ are almost degenerate and larger than $\sim 250 \mathrm{GeV}$. They are MSSM-like with a singlet admixture below $\sim 15$ percent. The combination of small phase space and not large enough couplings implies signal rates which are not in conflict with the present LHC exclusion limits. Note, that scans over larger ranges of the NMSSM parameter space lead to similar results.

In the future high-luminosity phase of the LHC with $14 \mathrm{TeV}$ c.m. energy it will be possible to find more than one Higgs boson eventually. In case three Higgs bosons will be found, precision measurements of couplings can be exploited to decide whether they are MSSM or NMSSM Higgs bosons. The NMSSM scalar Higgs boson couplings to gauge bosons fulfill the following sum rule,

$$
\sum_{i=1}^{3} g_{H_{i} V V}^{2}=1,
$$

and for the couplings to top and bottom quarks we have the sum rule

$$
\frac{1}{\sum_{i=1}^{3} g_{H_{i} t t}^{2}}+\frac{1}{\sum_{i=1}^{3} g_{H_{i} b b}^{2}}=1
$$

[in units of SM couplings]. In case only three NMSSM Higgs bosons are discovered, and not all of them are scalar, the measurement of their gauge and Yukawa couplings will show a violation of these sum rules due to the missing couplings of the non-discovered Higgs bosons. However, in the MSSM, with only three neutral Higgs bosons, the rules would be fulfilled. Figure 8 shows the result of a scan where $\mathrm{H}_{2}$ is SM-like and its rates are in accordance with the measured rates of the LHC experiments. It is supposed that only the two lightest CP-even Higgs bosons have been discovered. The left plot shows the violation of the vector coupling sum rule for $H_{1}$ and $H_{2}$, the right plot the violation of the Yukawa coupling sum rule. The respective sums deviate from 1 , in case of the fermion couplings by up to a factor of two. Figure 9 shows the same coupling sums but this time for the two heavier scalar bosons $H_{2}$ and $H_{3}$ and as a function of $M_{H_{1}}$, supposing that the lightest scalar Higgs boson has not been discovered due to too small SM couplings because of its singlet nature, but the heavier MSSM-like $H_{3}$ has been found. The measurement of the Yukawa couplings would show a violation of the sum rule by up to $\sim 20 \%$ in this case, the gauge couplings by up to $\sim 27 \%$. The largest deviations are observed when the lightest Higgs mass is of $\mathcal{O}(100 \mathrm{GeV})$, close to the $H_{2}$ mass, where the two states start to mix strongly. In both scenarios the determination of the couplings allows to distinguish the NMSSM Higgs sector from the MSSM provided that the precision in the coupling measurements is sufficiently high. 

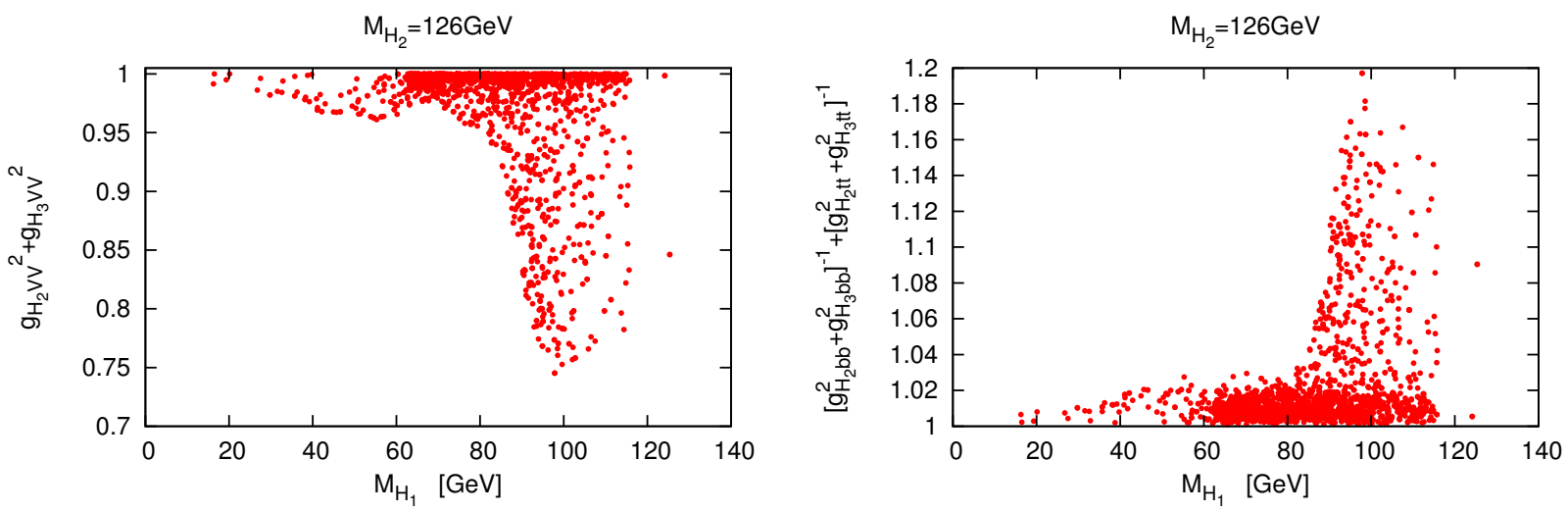

FIG. 9: Same as Fig. 8, but for $H_{2}$ (=SM-like) and $H_{3}$, and as a function of $M_{H_{1}}$.

In some scenarios, it may be possible to deduce the mass scale of the unobserved third CP-even scalar from the pattern of violation of the sum rules. For instance, Fig. 8 shows that larger deviations for the sum of the couplings of $H_{1}$ and $H_{2}$ to vector bosons are only possible for relatively small values of $M_{H_{3}}$, while maximal deviations of the fermion sum rule point towards larger $H_{3}$ masses. However, the figure also illustrates that very different values of $M_{H_{3}}$ can lead to very similar results for the sum rules, so that in general it is not possible to reliably infer bounds on the heavy scale $\left(M_{H_{3}}\right)$ from precision measurements of the light observed scalars. This can be understood from the fact that, contrary to the MSSM, the NMSSM at tree-level depends on four additional unknown parameters besides $\tan \beta$ and the charged Higgs boson mass $M_{H^{ \pm}}{ }^{\ddagger}$. These are given by the two NMSSM specific couplings $\lambda$ and $\kappa$, the vacuum expectation value $v_{s}$ of the singlet field and the soft SUSY breaking trilinear coupling $A_{\kappa}$. The additional parameters influence the values of the mixing angles and thus the Higgs boson couplings to the light scalar particles. Therefore there is no unique correlation between the coupling values and the scale of new physics, as given e.g. by the charged Higgs boson mass.

In the NMSSM the Higgs signal observed at the LHC could also be built up by two Higgs bosons, which are nearly degenerate in mass [91, 93, while such scenarios are difficult to achieve in the MSSM. In case no further Higgs bosons are discovered, the observation of a Higgs signal built up by two resonances clearly allows to distinguish the NMSSM from the SM case. The superposition of two (or more) nearly degenerate Higgs bosons near $126 \mathrm{GeV}$ can be tested experimentally by analyzing double ratios of signal rates [94]. The deviation from unity in this case could be tested at the $14 \mathrm{TeV}$ run of the LHC with high luminosity.

\section{LOOP EFFECTS}

Due to the numerical loop coefficient $1 /\left(16 \pi^{2}\right)$ and potentially small couplings, loop effects are less promising for probing energy scales with BSM physics, in particular because experimental analyses partly rely on tails of distributions. In general the mass range probed would be covered by direct searches for new particles at the LHC [and later LC]. Nevertheless, certain extensions of the Standard Model, such as supersymmetric models, generate parametrically enhanced loop corrections. In addition, somewhat exotic configurations, such as leptophilic particles equipped only with new $U(1)$ charges, may be suppressed in production modes at the LHC, and thus precision Higgs analyses may open a window to new BSM degrees of freedom.

\footnotetext{
$\ddagger$ The charged Higgs boson mass can be traded for the trilinear soft SUSY breaking coupling $A_{\lambda}$.
} 


\subsection{Simple examples}

Obviously, we cannot cover loop effects in all different Higgs production and decay modes, so that we focus on three representative cases: first, we discuss vertex corrections to fermionic Higgs decays, mediated by heavy gauge and scalar bosons. They are similar, in spirit, to the well studied corrections to $Z \rightarrow b \bar{b}$ decays in many models. Next, we introduce corrections to the loop-induced Higgs-gluon and Higgs-photon interactions, which benefit from the fact that the leading Standard Model amplitudes are already loop-suppressed. Finally, we show some distinctive effects of vector-like leptons.

\section{Heavy virtual bosons}

Higgs vertex corrections due to $Z^{\prime}$ vector boson and $\sigma^{\prime}$ scalar exchanges, as shown in Fig. 10, have a generic structure and a general magnitude of loop effects. In terms of the shift of the Higgs coupling to light fermions $g \rightarrow(1+\Delta) g$, one finds for a vector exchange:

$$
\Delta_{Z^{\prime}}=-\frac{g_{L} g_{R}}{4 \pi^{2}} m_{h}^{2} C_{0}\left(m_{h}^{2}, 0,0,0,0, m_{Z^{\prime}}^{2}\right) \simeq-\frac{g_{L} g_{R}}{4 \pi^{2}} \frac{m_{h}^{2}}{m_{Z^{\prime}}^{2}}\left[\log \frac{m_{h}^{2}}{m_{Z^{\prime}}^{2}}-1\right]
$$

where the last step corresponds to the limit $m_{Z^{\prime}} \gg m_{h}$. The couplings $g_{L / R}$ denote the left-chiral and rightchiral gauge couplings of the $Z^{\prime}$ boson to the light SM fermions.

To preserve gauge invariance, the scalar $\sigma^{\prime}$ must belong to an $S U(2)$ doublet $\phi^{\prime}$, but it is assumed not to be a Higgs boson i.e. not carry a vacuum expectation value. The leading correction to the $h b \bar{b}$ coupling reads, in the limit $m_{\phi^{\prime}} \gg m_{t} \gg m_{b}$ :

$$
\Delta_{\phi^{\prime}} \simeq \frac{y_{U} y_{D} y_{t}}{64 \pi^{2} y_{b}} \frac{m_{h}^{2}}{m_{\phi^{\prime}}^{2}}\left[4 \tau^{3} \arctan \frac{1}{\tau}-2 \tau^{2} \log \frac{m_{\phi^{\prime}}^{2}}{m_{t}^{2}}-2 \tau^{2}-1\right] \quad \text { with } \quad \tau=\left(4 m_{t}^{2} / m_{h}^{2}-1\right)^{1 / 2}
$$

where $y_{D, U}$ are the Yukawa couplings for the $\bar{q}_{L} \phi^{\prime} b_{R}$ and $\bar{q}_{L} \phi^{\prime c} t_{R}$ interactions, respectively. The result can be adapted straightforwardly for other fermion final states. We see that both corrections, Eq.4.1. and Eq.4.2. decrease with the square of the new physics mass scale, as expected qualitatively from the operator expansion and in accordance with the decoupling theorem [16]. Setting the couplings $g_{L} g_{R}=1\left(\right.$ or $\left.y_{U} y_{D} y_{t} / y_{b}=1\right)$, the magnitude of the corrections $\Delta$ is of order $10^{-2}$ or less for $m_{Z^{\prime}} \gtrsim 300 \mathrm{GeV}\left(m_{\phi^{\prime}} \gtrsim 300 \mathrm{GeV}\right)$, so significant deviations are expected only in exotic scenarios with large couplings.

\section{Loop-induced decays}

The effective Higgs-gluon and Higgs-photon couplings are excellent probes for new physics because already in the Standard Model they are loop-induced. The only difference between the top and $W$ loops in the Standard Model and new physics contributions is the actual mass suppression described in Eq. 2.13). While in $e^{+} e^{-}$ collisions Higgs decays to gluons can be extracted from the backgrounds, at the LHC the sensitive observable
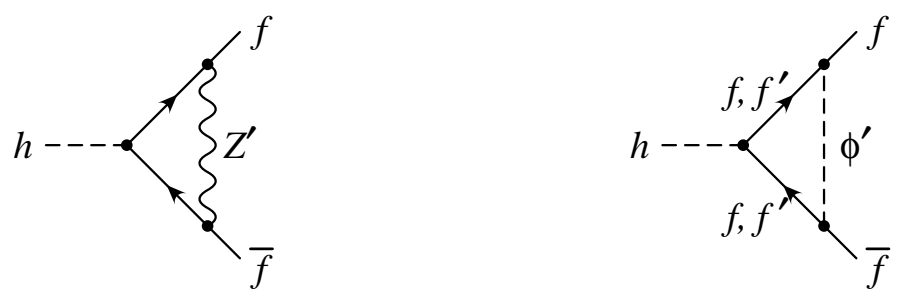

FIG. 10: Feynman diagrams for Higgs-fermion coupling corrections from vector boson or scalar exchange. 
to the Higgs-gluon coupling is the production cross section, as described in detail in Section 2 In the following we will focus on Higgs decays, but all results can be directly translated into the LHC production rate. Of course, the $h \rightarrow \gamma Z$ process is also a loop-induced interaction, but after including the $Z \rightarrow \ell^{+} \ell^{-}$branching fraction the expected event rate is very low, so it is challenging to precisely measure this channel at colliders. Therefore the $\gamma Z$ mode is in general less suitable for constraining generic new physics effects and thus will not be discussed here.

The SM results for loop-induced Higgs decays [95, 96, can be written as

$$
\begin{aligned}
\Gamma[h \rightarrow g g] & =\frac{\alpha_{\mathrm{s}}^{2} m_{h}^{3}}{128 \pi^{3}}\left|\mathcal{A}_{g g}\right|^{2}, \quad \mathcal{A}_{g g}=\left(\sqrt{2} G_{F}\right)^{1 / 2} \sum_{q} A_{1 / 2}\left(\tau_{q}\right), \\
\Gamma[h \rightarrow \gamma \gamma] & =\frac{\alpha^{2} m_{h}^{3}}{1024 \pi^{3}}\left|\mathcal{A}_{\gamma \gamma}\right|^{2}, \quad \mathcal{A}_{\gamma \gamma}=\left(4 \sqrt{2} G_{F}\right)^{1 / 2}\left[\sum_{f} N_{c}^{f} Q_{f}^{2} A_{1 / 2}\left(\tau_{f}\right)+A_{1}\left(\tau_{W}\right)\right],
\end{aligned}
$$

where $G_{F}$ is the Fermi constant, $N_{c}^{f}=1,3$ for leptons $(f=\ell)$ and quarks $(f=q)$, respectively, and $\tau_{i}=$ $4 m_{i}^{2} / m_{h}^{2}$. The loop functions are defined as

$$
\begin{aligned}
A_{1 / 2}(\tau) & =2 \tau[1+(1-\tau) f(\tau)], \\
A_{1}(\tau) & =-[2+3 \tau+3 \tau(2-\tau) f(\tau)],
\end{aligned}
$$

where

$$
f(\tau)= \begin{cases}\arcsin ^{2} \sqrt{1 / \tau}, & \tau \geq 1 \\ -\frac{1}{4}\left[\log \frac{1+\sqrt{1-\tau}}{1-\sqrt{1-\tau}}-i \pi\right]^{2}, & \tau<1\end{cases}
$$

A new heavy particle $X$ that couples to the Higgs boson with strength $g_{h X X}$ leads to an additional contribution to these rates given by [57, 97, 98 ]

$$
\begin{gathered}
\Delta \mathcal{A}_{g g}=\frac{g_{h X X}}{m_{X}^{2}} T_{X} \delta_{R} \begin{cases}A_{0}\left(\tau_{X}\right) & \text { for scalar } X, \\
2 m_{X} A_{1 / 2}\left(\tau_{X}\right) & \text { for fermion } X, \\
A_{1}\left(\tau_{X}\right) & \text { for vector } X,\end{cases} \\
\Delta \mathcal{A}_{\gamma \gamma}=\frac{g_{h X X}}{m_{X}^{2}} N_{c}^{X} Q_{X}^{2} \begin{cases}A_{0}\left(\tau_{X}\right) & \text { for scalar } X, \\
2 m_{X} A_{1 / 2}\left(\tau_{X}\right) & \text { for fermion } X, \\
A_{1}\left(\tau_{X}\right) & \text { for vector } X,\end{cases}
\end{gathered}
$$

where $T_{X}=0, \frac{1}{2}, 3$ and $N_{c}^{X}=1,3,8$ if $X$ is a QCD singlet, triplet or octet, respectively. Furthermore, $\delta_{R}=1 / 2$ for a self-conjugate field and $\delta_{R}=1$ otherwise, and

$$
A_{0}(\tau)=-\tau[1-\tau f(\tau)]
$$

Higher-order QCD corrections to the hgg interaction are known to be large, but they have been calculated to complete NNLO and partial NNNLO order [99] in the Standard Model within the heavy top quark limit, and to NLO for the generic new physics contributions in Eq. [4.6] [100] in the case of large loop particle masses. For estimating the sensitivity to heavy new physics effects, it is however sufficient to consider the tree-level formulae listed above, since bottom quark effects and mismatches of the relative QCD corrections between the different contributions range at the 10-20\% level.

For large values of $m_{X}$, the loop functions simplify to $A_{0}=\frac{1}{3}, A_{1 / 2}=\frac{4}{3}$, and $A_{1}=-7$. In this limit, the 
contributions in Eq. 4.3 can be mapped onto the $D=6$ operators in Table II according to

$$
\begin{gathered}
f_{G G}=-\frac{g_{h X X}}{96 \pi^{2} v} T_{X} \delta_{R} \begin{cases}1 & \text { for scalar } X, \\
8 m_{X} & \text { for fermion } X, \\
-21 & \text { for vector } X,\end{cases} \\
\frac{1}{2}\left(f_{B B}+f_{W W}-f_{B W}\right)=-\frac{g_{h X X}}{96 \pi^{2} v} N_{c}^{X} Q_{X}^{2} \begin{cases}1 & \text { for scalar } X, \\
8 m_{X} & \text { for fermion } X, \\
-21 & \text { for vector } X .\end{cases}
\end{gathered}
$$

Note that electroweak gauge symmetry demands that the coupling between $h$ and a fermion $X$ emerges from a dimension-5 operator of the form $\frac{1}{\Lambda} \phi^{\dagger} \phi \bar{X} X$, so that $g_{h X X}$ in this case should be of order $\mathcal{O}(v / \Lambda)$. Assuming a common new physics scale $\Lambda \sim m_{X}$, one can thus see that the expressions in eqs. 4.94 .10 are independent of $m_{X}$ for all spin assignments of $X$.

\section{Vector-like leptons}

An enlarged spectrum that feeds into modifications of the $h \rightarrow \gamma \gamma$ branching ratio is typically accompanied by a modification of the $h \rightarrow Z \gamma$ branching. While the effects on $h \rightarrow Z \gamma$ can be larger (e.g. in composite scenarios [101]), a measurement at the LHC can be challenging. A different avenue to formulate constraints on such a situation is via precision measurements at a future lepton collider. An enlarged spectrum modifies the $e^{+} e^{-} \rightarrow h Z$ production cross section through higher-order electroweak corrections, which can be significantly larger in the BSM theory. Such a cross section modification, which can be studied in a model-independent fashion at a future $250 \mathrm{GeV}$ lepton collider, might indeed be resolvable given the high precision measurements that can be performed with such a machine $102-104$. The uncertainties that arise at hadron colliders render such a measurement more difficult at the LHC, but nevertheless an important first step is possible. Depending on the precision of the coupling extraction that can be obtained at the LHC and the performance of, e.g., boosted analyses of $p p \rightarrow h Z$ production 38, deviations in the high $p_{T}$ region due to resolved loop contributions can be used to formulate bounds on non-SM contributions as discussed in [41].

In the following we will focus on these effects in the clean environment of a linear collider experiment, where they can be resolved best. Electroweak corrections are most straightforward in a well-defined framework. As a concrete model to review the precision measurement avenue we consider a simple scenario of vector-like leptons as discussed in 105 .

$$
\begin{aligned}
-\mathcal{L} & \supset m_{\ell} \bar{\ell}_{L}^{\prime} \ell_{R}^{\prime \prime}+m_{e} \bar{e}_{L}^{\prime \prime} e_{R}^{\prime}+m_{\nu} \bar{\nu}_{L}^{\prime \prime} \nu_{R}^{\prime}+\text { h.c. } \\
& +Y_{c}^{\prime}\left(\bar{\ell}_{L}^{\prime} h\right) e_{R}^{\prime}+Y_{n}^{\prime}\left(\bar{\ell}_{L}^{\prime} i \sigma^{2} h^{\dagger}\right) \nu_{R}^{\prime}+Y_{c}^{\prime \prime}\left(\bar{\ell}_{R}^{\prime \prime} h\right) e_{L}^{\prime \prime}+Y_{n}^{\prime \prime}\left(\bar{\ell}_{R}^{\prime \prime} i \sigma^{2} h^{\dagger}\right) \nu_{L}^{\prime \prime}+\text { h.c. }
\end{aligned}
$$

where $\ell_{L}^{\prime}, \ell_{R}^{\prime \prime}=(\mathbf{2},-1 / 2), e_{L}^{\prime \prime}, e_{R}^{\prime}=(\mathbf{1},-1)$, and $\nu_{L}^{\prime \prime}, \nu_{R}^{\prime}=(\mathbf{1}, 0)$ under $S U(2)_{L} \times U(1)_{Y}$. To reduce the number of free parameters we choose common values for the vector-like lepton masses

$$
m_{\ell}=m_{e}=m_{\nu}=m_{V}
$$

and common "chiral" masses from the couplings to the Higgs vev

$$
\begin{aligned}
& Y_{c}^{\prime} v / \sqrt{2}=Y_{c}^{\prime \prime} v / \sqrt{2}=m_{C h} \\
& Y_{n}^{\prime} v / \sqrt{2}=Y_{n}^{\prime \prime} v / \sqrt{2}=m_{C h}+\Delta_{\nu},
\end{aligned}
$$

in the following. Depending on these mass parameters [102, 103, 105], the $h \rightarrow \gamma \gamma$ branching fraction can be enhanced via Eq. 4.7), Fig. 11, while direct collider constraints and precision measurements are currently not sensitive to such a spectrum (see Ref. [105] for a discussion). Obviously the potentially large enhancement of the $h \rightarrow \gamma \gamma$ branching ratio is a way to constrain the chiral component of this model at the LHC once the $h \rightarrow \gamma \gamma$ becomes SM-like. However, the percent-level precision measurement of the associated production cross section at a linear collider can supersede the LHC measurement as indicated in Fig. 11. 

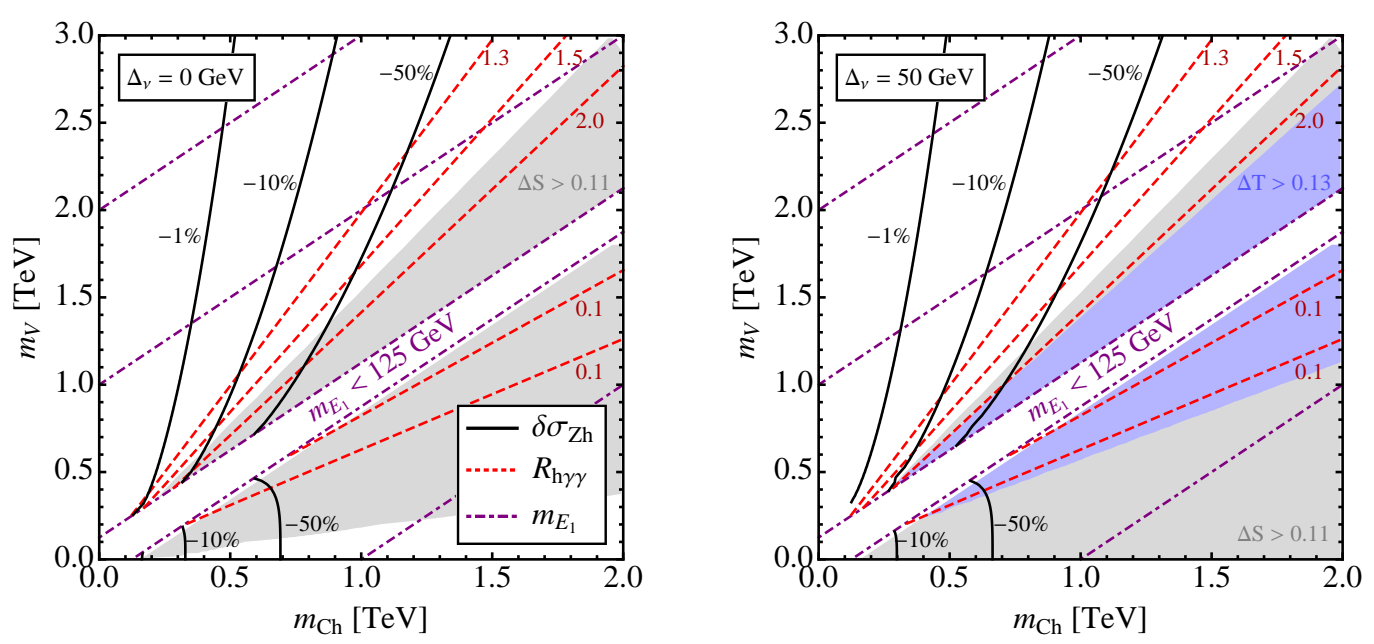

FIG. 11: Correlation of the diphoton branching modification (red dashed) and the modification of the associated Higgs production cross section (solid black) that arises as part of the NLO electroweak correction. Parameter regions that are excluded by oblique correction constraints on $\Delta T, \Delta S$ are shaded. The parameter region that results in a lightest charged lepton eigenvalue $E_{1}$ lighter than $125 \mathrm{GeV}$ is excluded. The left plot corresponds to $\Delta_{\nu}=0$, while in the right plot $\Delta_{\nu}=50 \mathrm{GeV}$. Figure taken from Ref. [103].

Contextualizing Fig. 11 with the aim of our review, we can identify a region $m_{C h} \sim v$ in 4.13 and make a prediction on the vectorial mass terms (the new mass scale in this concrete model) given a precision percentlevel measurement at a future lepton collider. Figure 11 shows that the new physics scale is in this case at around $1.5 \mathrm{TeV}$.

\section{2. $\tan \beta$-enhanced non-decoupling effects}

While generic effects of weakly interacting extended Higgs sectors can be expected to decouple with the mass scale of the heavy new states, this does not have to be true for all models. In general extended Higgs models there exists a universal source of such effects: the mass of the heavy new state might receive contributions both directly from the Higgs potential Eq. 3.10) and from a combination of a vev and a self interaction, $m_{H^{0}}^{2} \sim m_{12}^{2}+\lambda_{3} v_{1}^{2} / 2$. The second term appears as a result of spontaneous symmetry breaking and avoids the Appelquist-Carazzone decoupling theorem [16] when $v_{1}$ and thus $m_{H^{0}}$ become large. It can lead to substantial corrections to the effective quartic Higgs coupling, but also to the other Higgs couplings. More generally, large contributions of the kind $\lambda_{i} v^{2}$ can induce unexpectedly large effects, because they are only constrained by the high-scale behavior of the extended Higgs model.

In general Two-Higgs-Doublet models the most dangerous source of large quantum corrections is related to the form of the top Yukawa coupling to the heavy states: dependent on the model setup it scales with $m_{t} / \tan \beta$, which means that for $\tan \beta<1$ it rapidly approaches a Landau pole. Experimentally, such effects are strongly constrained for example by $B_{d}-\bar{B}_{d}$ mixing.

In the type-II 2HDM of the MSSM, a subset of the radiative corrections to down-type Yukawa couplings, $y_{d}$, are enhanced by $\tan \beta$ and do not decouple in the limit $\mu \sim M_{\mathrm{SUSY}} \gg v$ 106. These corrections emerge from loop contributions to the down-type quark-Higgs coupling involving the insertion of the up-type Higgs field $\phi_{2}$ instead of the down-type Higgs field $\phi_{1}$, see Fig. 12 (a). This calculation can be reduced to the corresponding self-energies with the insertion of the vev $v_{2}$ and using its relation to the full Higgs field $\phi_{2}$ by means of a low-energy theorem [95]. The leading terms are proportional to the QCD coupling $\alpha_{\mathrm{s}} \tan \beta$ or the top Yukawa coupling $y_{t} \tan \beta$ and thus can be $\mathcal{O}(1)$. For the $h^{0} b \bar{b}$ coupling they can be written, in the limit $\mu \sim M_{\mathrm{SUSY}} \gg v$, 
(a)

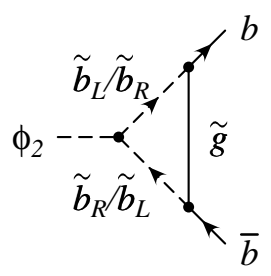

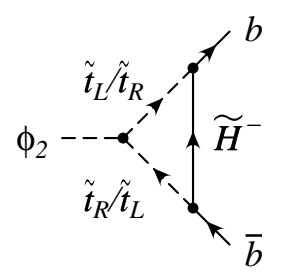

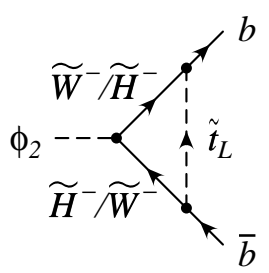

(b)

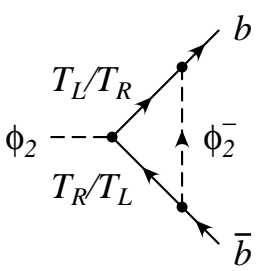

FIG. 12: Feynman diagrams leading to $\tan \beta$-enhanced corrections to the bottom-quark Yukawa coupling in (a) the MSSM and (b) the model 4.15 with heavy vector-like fermions. Here $\phi_{2}$ is the second Higgs doublet, and $\tilde{b}, \tilde{t}, \tilde{g}, \tilde{W}^{-}$, and $\tilde{H}^{-}$are the sbottom, stop, gluino, wino and higgsino fields, respectively.

as

$$
\begin{aligned}
& \frac{\Gamma_{\mathrm{SUSY}}\left[h^{0} \rightarrow b b\right]}{\Gamma_{\mathrm{Born}}\left[h^{0} \rightarrow b b\right]}=\left[\frac{1}{1+\Delta_{b}}\left(1-\frac{\Delta_{b}}{\tan \alpha \tan \beta}\right)\right]^{2}, \\
& \Delta_{b}=-\frac{2 \alpha_{\mathrm{s}} \tan \beta}{3 \pi} \frac{\mu}{m_{\tilde{g}}} I\left(\frac{m_{\tilde{b}_{L}}^{2}}{m_{\tilde{g}}^{2}}, \frac{m_{\tilde{b}_{R}}^{2}}{m_{\tilde{g}}^{2}}\right)-\frac{y_{t}^{2} \tan \beta}{16 \pi^{2}} \frac{A_{t}}{\mu} I\left(\frac{m_{\tilde{t}_{L}}^{2}}{\mu^{2}}, \frac{m_{\tilde{t}_{R}}^{2}}{\mu^{2}}\right)+\frac{3 g^{2} \tan \beta}{32 \pi^{2}} \frac{M_{2}}{\mu} I\left(\frac{m_{\tilde{t}_{L}}^{2}}{\mu^{2}} \frac{M_{2}^{2}}{\mu^{2}}\right) \\
& I(x, y)=\frac{x \log x}{(1-x)(x-y)}+\frac{y \log y}{(1-y)(y-x)} .
\end{aligned}
$$

Here $\mu$ and $M_{2}$ are the higgsino and wino mass parameters, respectively; $m_{\tilde{b}_{L, R}}, m_{\tilde{t}_{L, R}}$ and $m_{\tilde{g}}$ denote the masses of the sbottoms, stops and gluino, respectively; and $y_{t}$ is the top Yukawa coupling. Note that for the leading contribution in $\tan \beta$, mixing between the $\mathrm{L}$ - and $\mathrm{R}$-sfermions and among the charginos can be neglected.

Besides the terms listed in Eq. 4.14 there are additional contributions proportional to $g^{\prime 2}$ which, however, are suppressed by the small hypercharge of the bottom quark and thus negligible. The leading two-loop corrections to these effective Yukawa couplings have been determined in Ref. 107. They are of moderate size if the scales of the QCD coupling $\alpha_{s}$ and the top Yukawa coupling $y_{t}$ are chosen as the average of the correspondingly contributing SUSY masses.

While the occurrence of non-decoupling $\tan \beta$-enhanced corrections is natural in the MSSM or other supersymmetric models [108, they can more generally appear in a large class of models that include the 2HDM type-II and a set of new particles at the mass scale $\Lambda$. The crucial condition is that large mixing between the up- and down-type Yukawa sectors is generated through loops of $\mathcal{O}(\Lambda)$ fields. [In the MSSM this mixing is achieved by the $\mu$ term.]

For example, we may consider a type-II $2 \mathrm{HDM}$ supplemented by a dark (hidden) sector that is charged under a $\mathbb{Z}_{2}$ symmetry. The dark sector contains vector-like fermionic partners of the top quark, denoted $Q_{L}=\left(T_{L}, B_{L}\right)$ and $T_{R}$, as well as a scalar partner of the SM-like Higgs doublet $\phi_{1}$, denoted $\phi_{2}$, with Yukawa couplings of the form

$$
\mathcal{L}_{\text {dark }, \text { yuk }}=-x_{d} y_{b} \bar{Q}_{L} \phi_{2} b_{R}-x_{u} y_{t} \bar{q}_{3 L} \phi_{2}^{c} T_{R}-y_{t} \bar{Q}_{L} \phi_{1}^{c} T_{R}+\text { h.c. },
$$

where $q_{3 L}=\left(t_{L}, b_{L}\right)$ is the left-handed SM quark doublet, and $x_{u}$ and $x_{d}$ are arbitrary $\mathcal{O}(1)$ parameters. It is assumed that the fields $Q_{L}, T_{R}$ and $\phi_{2}$ all have masses $m_{Q_{L}} \sim m_{T_{R}} \sim m_{\phi_{2}} \sim \Lambda$. The dark sector may contain additional fields, but they are unnecessary for our purposes. Since $\phi_{2}$ couples to both up- and down-type quarks in eq. 4.15, it generates the required mixing between these two sectors. As a result, the loop diagram involving $T_{L}, T_{R}$, and the charged component of $\phi_{2}$, see Fig. 12 (b), leads to the $\tan \beta$-enhanced correction

$$
\Delta_{b}=\frac{y_{t}^{2} x_{u} x_{d} \tan \beta}{4 \pi^{2}}+\mathcal{O}\left(\frac{v^{2}}{\Lambda^{2}}\right)
$$

Generically, therefore, a large deviation with respect to the Standard Model, of the Higgs couplings to down-type fermions may be interpreted as a hint for the presence of a second Higgs doublet and a hidden sector. 


\begin{tabular}{|l||c|c||c|c|}
\hline Scenario/framework & LHC & HL-LHC & LC & HL-LC \\
\hline \hline Higgs portal & 0.23 & 0.28 & 0.44 & 0.56 \\
2 HDM type-II $(\tan \beta \approx 1)$ & 0.52 & 0.58 & 1.15 & 1.6 \\
2 HDM type-II $(\tan \beta \approx 10)$ & 0.33 & 0.36 & 0.7 & 1.0 \\
\hline$D=6$ effective operators: & & & & \\
$h V V$ & 0.78 & 0.87 & 2.6 & 3.3 \\
$h f f$ & 0.45 & 0.50 & 1.0 & 1.4 \\
$h g g$ contact & 0.55 & 1.1 & 1.3 & 1.8 \\
$h \gamma \gamma$ contact & 0.15 & 0.18 & 0.24 & 0.36 \\
\hline Strong interactions & 0.9 & $1.1-2.0$ & $2.8-5.1$ & $3.4-5.6$ \\
\hline$h g g$ loop effects: & & & & \\
scalar triplet & 0.16 & 0.31 & 0.37 & 0.52 \\
scalar octet & 0.39 & 0.75 & 0.92 & 1.3 \\
vector octet & 1.8 & 3.5 & 4.2 & 5.8 \\
$h \gamma \gamma$ loop effects: & & & & \\
scalar triplet & 0.15 & 0.18 & 0.24 & 0.36 \\
scalar octet & 0.25 & 0.29 & 0.39 & 0.60 \\
vector octet & 1.1 & 1.3 & 1.8 & 2.7 \\
\hline Vector-like leptons & - & - & 1.2 & 1.5 \\
\hline
\end{tabular}

TABLE VI: Effective sensitivity to new physics scales (in TeV) at the level of one standard deviation from measurements of the Higgs couplings at the LHC and a future LC (with different assumptions about the luminosity, see Table I) in a variety of BSM models.

\section{SUMMARY AND CONCLUSIONS}

The discovery of the Higgs boson not only concludes the search for the fundamental degrees of freedom predicted by the Standard Model, but also provides us with the unique opportunity to gain information about anticipated new physics through a detailed study of its properties. We have evaluated the sensitivity of Higgs precision measurements to various new physics scenarios. The main findings are summarized in TableVI, based on the following input:

- Higgs portal: Eq. (3.7) combined with Table I

- Two-Higgs-Doublet Model: Eq.(3.13) combined with Table I]

- effective dimension-6 operators: Results from Table III

- Strongly interacting Higgs field: Results from Table IV

- Heavy virtual bosons: No competitive limits;

- Loop-induced decays: Eqs. 4.67, 4.7) with $\delta_{R}=1, Q_{X}=1$ and Table I

- Vector-like leptons: Fig. 11 for $m_{C h} \approx v$.

In deriving the limits quoted in the table, we have generically assumed that the couplings between the Higgs boson and the new physics sector are $\mathcal{O}(1)$ for gauge-invariant dimensionless couplings or $\mathcal{O}(v)$ for couplings with mass dimension one.

Evidently, Higgs precision data can be sensitive to multi-TeV scales, beyond the reach of direct LHC searches as well as electroweak precision tests. This is true in particular if the new physics (i) modifies the Higgs couplings to $W$ and $Z$ bosons, (ii) is related to strong dynamics, or (iii) involves new heavy vector bosons that generate loop corrections to the Higgs-gluon or Higgs-photon interactions. On the other hand, in minimal weakly 
coupled scenarios such as the Higgs portal or a scalar triplet loop in Higgs-gluon or Higgs-photon couplings, the Higgs coupling measurements only probe sub-TeV scales, and thus complement direct LHC searches.

Observation of significant deviations in future Higgs coupling precision measurements, combined with data from direct searches for new particles at the LHC and precision electroweak and flavor constraints, will allow one to narrow down the possibilities for new physics that can explain the Higgs coupling shifts. The pattern of deviations in the different couplings carries additional information. For instance, extended scalar sectors may lead to large deviations in the Higgs-fermion couplings, as illustrated in eq. (3.13) and in Figs. 8. On the other hand, sizeable shifts in the Higgs-gauge boson couplings can be generated, for instance, by strong interactions.

Most notably, in supersymmetric theories and models with similar particle content, there can be large radiative corrections to the coupling between the Higgs boson and down-type quarks that do not decouple for large values of the mass scale of the particles in the loop. Such a scenario offers sensitivity to extremely high scales from Higgs precision physics, although it may be fine-tuned from a model building perspective.

If multiple Higgs scalars are directly observed at the LHC, the measurement of their couplings will again yield valuable information about potential extra unobserved states. In particular, the discovery of three neutral Higgs bosons would suggest a Two-Higgs-Doublet model as the underlying theory, as for example in the MSSM, but they may also be part of a more complex scalar sector, involving for instance an additional singlet, as in the NMSSM. These two cases may be distinguished by analyzing the couplings of the three observed states to gauge bosons and fermions, which would violate certain sum rules if the additional particles are not completely decoupled. However, the mass scale of the extra scalars (beyond the two Higgs doublets) can in general not be deduced from this information, since any extension of the Two-Higgs-Doublet model introduces several new parameters, which may lead to partial cancellations in the corrections to the couplings.

In either of these two scenarios, with only one observed Higgs boson or multiple Higgs bosons at the LHC, precision measurements of its couplings provide a unique window into possible new physics sectors, which are not excluded by current data. In order to fully exploit this avenue, percent-level precision for the determination of Higgs production and decay rates will be essential, a goal that can be achieved by a future high-energy $e^{+} e^{-}$ collider, such as the ILC.

\section{Acknowledgements}

The authors are deeply indebted to P. M. Zerwas, who has initiated this review and has provided extensive advise during its preparation. We also thank C. Grojean and A. Pomarol for helpful discussions. C.E. is supported in parts by the IPPP Associateship programme. A.F. acknowledges support by the National Science Foundation under grant no. PHY-1212635. M.M., M.R. and K.W. are supported by the DFG SFB/TR9 "Computational Particle Physics". M.R. additionally acknowledges support by the BMBF under Grant No. 05H09VKG ("Verbundprojekt HEP-Theorie").

[1] P. W. Higgs, Phys. Lett. 12, 132 (1964), Phys. Rev. Lett. 13, 508 (1964) and Phys. Rev. 145, 1156 (1964); F. Englert and R. Brout, Phys. Rev. Lett. 13, 321 (1964); G.S. Guralnik, C.R. Hagen and T.W. Kibble, Phys. Rev. Lett. 13, 585 (1964).

[2] G. Aad et al. [ATLAS Collaboration], Phys. Lett. B 716, 1 (2012); S. Chatrchyan et al. [CMS Collaboration], Phys. Lett. B 716, 30 (2012) and JHEP 1306, 081 (2013).

[3] H. Murayama, arXiv:0704.2276 [hep-ph]; Y. Gershtein et al., arXiv:1311.0299 [hep-ex].

[4] See e.g. M. Shaposhnikov and C. Wetterich, Phys. Lett. B 683, 196 (2010); M. Holthausen, K. S. Lim and M. Lindner, JHEP 1202, 037 (2012); A. Hebecker, A. K. Knochel and T. Weigand, Nucl. Phys. B 874, 1 (2013); D. Buttazzo et al, JHEP 1312, 089 (2013).

[5] For pedagogical introductions and reviews see e.g. M. Spira and P. M. Zerwas, Lect. Notes Phys. 512, 161 (1998); M. Gomez-Bock, M. Mondragon, M. Mühlleitner, R. Noriega-Papaqui, I. Pedraza, M. Spira and P. M. Zerwas, 
J. Phys. Conf. Ser. 18, 74 (2005); M. Gomez-Bock, M. Mondragon, M. Mühlleitner, M. Spira and P. M. Zerwas, arXiv:0712.2419 [hep-ph]; M. Spira, Fortsch. Phys. 46, 203 (1998); A. Djouadi, Phys. Rept. 457, 1 (2008); T. Plehn, Lect. Notes Phys. 844, 1 (2012).

[6] G. Aad et al. [ATLAS Collaboration], ATLAS-CONF-2013-013, ATLAS-CONF-2013-034, ATLAS-CONF-2013040; S. Chatrchyan et al. [CMS Collaboration], CMS-PAS-HIG-13-005, arXiv:1312.5353 [hep-ex].

[7] For a discussion of angular observables see e.g. E.P. Shabalin, Zh. Eksperim. i Teor. Fiz. 44, 765 (1963) JETP 17, 517 (1963); N. Cabibbo and A. Maksymowicz, Phys. Rev. 137 (1965) B438 [Erratum-ibid. 168, 1926 (1968)]; T. L. Trueman, Phys. Rev. D 18, 3423 (1978); J. R. Dell'Aquila and C. A. Nelson, Phys. Rev. D 33, 80 (1986); S. Y. Choi, D. J. Miller, M. M. Mühlleitner and P. M. Zerwas, Phys. Lett. B 553, 61 (2003); Y. Gao, A. V. Gritsan, Z. Guo, K. Melnikov, M. Schulze and N. V. Tran, Phys. Rev. D 81, 075022 (2010); C. Englert, C. Hackstein and M. Spannowsky, Phys. Rev. D 82, 114024 (2010); J. Ellis and D. S. Hwang, JHEP 1209, 071 (2012); S. Y. Choi, M. M. Mühlleitner and P. M. Zerwas, Phys. Lett. B 718, 1031 (2013).

[8] T. Plehn, D. L. Rainwater and D. Zeppenfeld, Phys. Rev. Lett. 88, 051801 (2002); C. Ruwiedel, N. Wermes and M. Schumacher, Eur. Phys. J. C 51, 385 (2007); K. Hagiwara, Q. Li and K. Mawatari, JHEP 0907, 101 (2009); Y. Gao, A. V. Gritsan, Z. Guo, K. Melnikov, M. Schulze and N. V. Tran, Phys. Rev. D 81, 075022 (2010); C. Englert, M. Spannowsky and M. Takeuchi, JHEP 1206, 108 (2012); J. Ellis, D. S. Hwang, V. Sanz and T. You, JHEP 1211, 134 (2012); C.-Q. Geng, D. Huang, Y. Tang and Y.-L. Wu, Phys. Lett. B 719, 164 (2013); J. Ellis, R. Fok, D. S. Hwang, V. Sanz and T. You, Eur. Phys. J. C 73, 2488 (2013); J. Frank, M. Rauch and D. Zeppenfeld, Phys. Rev. D87, 055020 (2013); C. Englert, D. Goncalves-Netto, K. Mawatari and T. Plehn, JHEP 1301, 148 (2013); S. Banerjee, J. Kalinowski, W. Kotlarski, T. Przedzinski and Z. Was, Eur. Phys. J. C 73, 2313 (2013); A. Djouadi, R. M. Godbole, B. Mellado and K. Mohan, Phys. Lett. B 723, 307 (2013); C. Englert, D. Goncalves, G. Nail and M. Spannowsky, Phys. Rev. D 88, 013016 (2013); D. Boer, W. J. d. Dunnen, C. Pisano and M. Schlegel, Phys. Rev. Lett. 111, 032002 (2013); J. Frank, M. Rauch and D. Zeppenfeld, arXiv:1305.1883 [hep-ph]; J. Gao, JHEP 1402, 094 (2014); A. Kobakhidze and J. Yue, Phys. Lett. B 727, 456 (2013).

[9] R. Lafaye, T. Plehn, M. Rauch, D. Zerwas and M. Dührssen, JHEP 0908, 009 (2009); M. Klute, R. Lafaye, T. Plehn, M. Rauch and D. Zerwas, Phys. Rev. Lett. 109, 101801 (2012); T. Plehn and M. Rauch, Europhys. Lett. 100, $11002(2012)$.

[10] P. Bechtle, S. Heinemeyer, O. Stal, T. Stefaniak and G. Weiglein, arXiv:1403.1582 [hep-ph].

[11] A. Azatov, R. Contino and J. Galloway, JHEP 1204, 127 (2012); P. P. Giardino, K. Kannike, M. Raidal and A. Strumia, Phys. Lett. B 718, 469 (2012); J. Ellis and T. You, JHEP 1209, 123 (2012); J. R. Espinosa, C. Grojean, M. Mühlleitner and M. Trott, JHEP 1205, 097 (2012), JHEP 1209, 126 (2012) 126, and JHEP 1212, 045 (2012); A. Djouadi and G. Moreau, arXiv:1303.6591 [hep-ph]; J. Ellis and T. You, JHEP 1306, 103 (2013).

[12] E. Masso and V. Sanz, Phys. Rev. D 87, 033001 (2013).

[13] M. Klute, R. Lafaye, T. Plehn, M. Rauch and D. Zerwas, Europhys. Lett. 101, 51001 (2013).

[14] J. A. Aguilar-Saavedra et al. [ECFA/DESY LC Physics Working Group Collaboration], hep-ph/0106315; E. Accomando et al. [CLIC Physics Working Group Collaboration], hep-ph/0412251; G. Aarons et al. [ILC Collaboration], arXiv:0709.1893 [hep-ph]; H. Baer et al., arXiv:1306.6352 [hep-ph].

[15] B. A. Dobrescu and J. D. Lykken, JHEP 1302, 073 (2013).

[16] T. Appelquist and J. Carazzone, Phys. Rev. D 11, 2856 (1975).

[17] C. J. C. Burges and H. J. Schnitzer, Nucl. Phys. B 228, 464 (1983); C. N. Leung, S. T. Love and S. Rao, Z. Phys. C 31, 433 (1986); W. Buchmüller and D. Wyler, Nucl. Phys. B 268, 621 (1986); for a recent review on definitions and tools see D. Wackeroth (ed.) et al., arXiv:1309.7890 [hep-ph].

[18] B. Grzadkowski, M. Iskrzynski, M. Misiak and J. Rosiek, JHEP 1010, 085 (2010).

[19] D. Lopez-Val, T. Plehn and M. Rauch, JHEP 1310, 134 (2013).

[20] P. H. Frampton, P. Q. Hung and M. Sher, Phys. Rept. 330, 263 (2000); G. D. Kribs, T. Plehn, M. Spannowsky and T. M. P. Tait, Phys. Rev. D 76, 075016 (2007); O. Eberhardt, G. Herbert, H. Lacker, A. Lenz, A. Menzel, U. Nierste and M. Wiebusch, Phys. Rev. Lett. 109, 241802 (2012).

[21] C. H. Llewellyn Smith, Phys. Lett. B 46, 233 (1973); J. M. Cornwall, D. N. Levin and G. Tiktopoulos, Phys. Rev. D 10, 1145 (1974) [Erratum-ibid. D 11, 972 (1975)]; G. Passarino, Nucl. Phys. B 868, 416 (2013).

[22] T. D. Lee, Phys. Rev. D 8, 1226 (1973); R. A. Flores and M. Sher, Annals Phys. 148, 95 (1983); J. F. Gunion, H. E. Haber, G. L. Kane and S. Dawson, The Higgs hunter's guide, Addison-Wesley, Menlo-Park, 1990; G. C. Branco, P. M. Ferreira, L. Lavoura, M. N. Rebelo, M. Sher and J. P. Silva, Phys. Rept. 516, 1 (2012).

[23] J. F. Gunion and H. E. Haber, Phys. Rev. D 67, 075019 (2003).

[24] T. Binoth and J. J. van der Bij, Z. Phys. C 75, 17 (1997); B. Patt and F. Wilczek, hep-ph/0605188. 
[25] R. Schabinger and J. D. Wells, Phys. Rev. D 72, 093007 (2005).

[26] S. Bock, R. Lafaye, T. Plehn, M. Rauch, D. Zerwas and P. M. Zerwas, Phys. Lett. B 694, 44 (2010); C. Englert, T. Plehn, D. Zerwas and P. M. Zerwas, Phys. Lett. B 703, 298 (2011); C. Englert, T. Plehn, M. Rauch, D. Zerwas and P. M. Zerwas, Phys. Lett. B 707, 512 (2012).

[27] J. H. Collins and J. D. Wells, arXiv:1210.0205 [hep-ph].

[28] O. J. P. Eboli and D. Zeppenfeld, Phys. Lett. B 495, 147 (2000); C. Englert, J. Jaeckel, E. Re and M. Spannowsky, Phys. Rev. D 85, 035008 (2012); C. Englert, M. Spannowsky and C. Wymant, Phys. Lett. B 718, 538 (2012); D. Curtin et al., arXiv:1312.4992 [hep-ph].

[29] R. Contino, Y. Nomura and A. Pomarol, Nucl. Phys. B 671, 148 (2003).

[30] K. Agashe, R. Contino and A. Pomarol, Nucl. Phys. B 719, 165 (2005).

[31] R. Contino, L. Da Rold and A. Pomarol, Phys. Rev. D 75, 055014 (2007).

[32] J. R. Espinosa, C. Grojean and M. Mühlleitner, JHEP 1005, 065 (2010).

[33] D. Zeppenfeld, R. Kinnunen, A. Nikitenko and E. Richter-Was, Phys. Rev. D 62, 013009 (2000); M. Dührssen, S. Heinemeyer, H. Logan, D. Rainwater, G. Weiglein and D. Zeppenfeld, Phys. Rev. D 70, 113009 (2004); M. Dührssen, ATL-PHYS-2003-030.

[34] G. Aad et al. [ATLAS Collaboration], Phys. Lett. B 726, 88 (2013); S. Chatrchyan et al. [CMS Collaboration], JHEP 1401, 096 (2014).

[35] R. K. Ellis, I. Hinchliffe, M. Soldate and J. J. van der Bij, Nucl. Phys. B 297, 221 (1988).

[36] D. L. Rainwater, D. Zeppenfeld and K. Hagiwara, Phys. Rev. D 59, 014037 (1998); T. Plehn, D. L. Rainwater and D. Zeppenfeld, Phys. Rev. D 61, 093005 (2000).

[37] S. Chatrchyan et al. [CMS Collaboration], arXiv:1401.5041 [hep-ex]; G. Aad et al. [ATLAS Collaboration], ATLASCONF-2013-108.

[38] J. M. Butterworth, A. R. Davison, M. Rubin and G. P. Salam, Phys. Rev. Lett. 100, 242001 (2008); D. E. Soper and M. Spannowsky, JHEP 1008, 029 (2010); T. Plehn, P. Schichtel and D. Wiegand, arXiv:1311.2591 [hep-ph].

[39] S. Chatrchyan et al. [CMS Collaboration], Phys. Rev. D 89, 012003 (2014); G. Aad et al. [ATLAS Collaboration], ATLAS-CONF-2013-079.

[40] V. Drollinger, T. Müller and D. Denegri, hep-ph/0111312; J. Cammin and M. Schumacher, ATL-PHYS-2003-024; F. Maltoni, D. L. Rainwater and S. Willenbrock, Phys. Rev. D 66, 034022 (2002); T. Plehn, G. P. Salam and M. Spannowsky, Phys. Rev. Lett. 104, 111801 (2010); P. Artoisenet, P. de Aquino, F. Maltoni and O. Mattelaer, Phys. Rev. Lett. 111, 091802 (2013); D. Curtin, J. Galloway and J. G. Wacker, Phys. Rev. D 88, 093006 (2013); P. Agrawal, S. Bandyopadhyay and S. P. Das, Phys. Rev. D 88, 093008 (2013); M. R. Buckley, T. Plehn, T. Schell and M. Takeuchi, JHEP 1402, 130 (2014).

[41] A. Banfi, A. Martin and V. Sanz, arXiv:1308.4771 [hep-ph]; A. Azatov and A. Paul, JHEP 1401, 014 (2014); C. Englert, M. McCullough and M. Spannowsky, Phys. Rev. D 89, 013013 (2014); C. Grojean, E. Salvioni, M. Schlaffer and A. Weiler, arXiv:1312.3317 [hep-ph].

[42] M. Baak et al., Eur. Phys. J. C 72, 2003 (2012), updates available at http://gfitter.desy.de S. Schael et al. [ALEPH and DELPHI and L3 and OPAL and LEP Electroweak Working Group Collaborations], Phys. Rept. 532, 119 (2013).

[43] F. Bonnet, M. B. Gavela, T. Ota and W. Winter, Phys. Rev. D 85, 035016 (2012); F. Bonnet, T. Ota, M. Rauch and W. Winter, Phys. Rev. D 86, 093014 (2012).

[44] T. Corbett, O. J. P. Eboli, J. Gonzalez-Fraile and M. C. Gonzalez-Garcia, Phys. Rev. D 86, 075013 (2012); T. Corbett, O. J. P. Eboli, J. Gonzalez-Fraile and M. C. Gonzalez-Garcia, Phys. Rev. D 87, 015022 (2013).

[45] B. Dumont, S. Fichet and G. von Gersdorff, JHEP 1307, 065 (2013); M. B. Einhorn and J. Wudka, Nucl. Phys. B 877, 792 (2013); E. Boos, V. Bunichev, M. Dubinin and Y. Kurihara, Phys. Rev. D 89, 035001 (2014); S. Banerjee, S. Mukhopadhyay and B. Mukhopadhyaya, Phys. Rev. D 89, 053010 (2014).

[46] For an overview see e.g. I. Brivio et al., arXiv:1311.1823 [hep-ph].

[47] M. E. Peskin and T. Takeuchi, Phys. Rev. Lett. 65, 964 (1990); G. Altarelli and R. Barbieri, Phys. Lett. B 253, 161 (1991); G. Altarelli, R. Barbieri and S. Jadach, Nucl. Phys. B 369, 3 (1992) [Erratum ibid. B 376, 444 (1992)]; G. Altarelli, R. Barbieri and F. Caravaglios, Nucl. Phys. B 405, 3 (1993).

[48] K. Hagiwara, S. Ishihara, R. Szalapski and D. Zeppenfeld, Phys. Rev. D 48, 2182 (1993); H. Mebane, N. Greiner, C. Zhang and S. Willenbrock, Phys. Rev. D 88, 015028 (2013).

[49] R. Barbieri, A. Pomarol, R. Rattazzi and A. Strumia, Nucl. Phys. B 703, 127 (2004).

[50] A. De Rujula, M. B. Gavela, P. Hernandez and E. Masso, Nucl. Phys. B 384, 3 (1992); J. Elias-Miro, J. R. Espinosa, E. Masso and A. Pomarol, JHEP 1311, 066 (2013). 
[51] A. Pomarol and F. Riva, JHEP 1401, 151 (2014).

[52] R. Contino, M. Ghezzi, C. Grojean, M. Mühlleitner and M. Spira, JHEP 1307, 035 (2013) and arXiv:1403.3381 [hep-ph].

[53] R. N. Cahn, M. S. Chanowitz and N. Fleishon, Phys. Lett. B 82, 113 (1979); L. Bergström and G. Hulth, Nucl. Phys. B 259, 137 (1985) [Erratum-ibid. B 276, 744 (1986)]; M. Spira, A. Djouadi and P. M. Zerwas, Phys. Lett. B 276, 350 (1992).

[54] L. Lavoura and J. P. Silva, Phys. Rev. D 47, 2046 (1993).

[55] P. Lodone, JHEP 0812, 029 (2008); M. Gillioz, Phys. Rev. D 80, 055003 (2009); C. Anastasiou, E. Furlan and J. Santiago, Phys. Rev. D 79, 075003 (2009).

[56] C. Grojean, O. Matsedonskyi and G. Panico, JHEP 1310, 160 (2013).

[57] M. Gillioz, R. Gröber, A. Kapuvari and M. Mühlleitner, JHEP 1403, 037 (2014).

[58] A. Hayreter and G. Valencia, Phys. Rev. D 88, 034033 (2013).

[59] G. F. Giudice, C. Grojean, A. Pomarol and R. Rattazzi, JHEP 0706, 045 (2007).

[60] R. Gröber and M. Mühlleitner, JHEP 1106, 020 (2011).

[61] R. Contino, C. Grojean, M. Moretti, F. Piccinini and R. Rattazzi, JHEP 1005, 089 (2010).

[62] R. Contino, C. Grojean, D. Pappadopulo, R. Rattazzi and A. Thamm, JHEP 1402, 006 (2014).

[63] M. J. Dolan, C. Englert and M. Spannowsky, Phys. Rev. D 87, 5 (2013), 055002.

[64] V. Barger, T. Han, P. Langacker, B. McElrath and P. Zerwas, Phys. Rev. D 67, 115001 (2003).

[65] K. Hagiwara, R. Szalapski and D. Zeppenfeld, Phys. Lett. B 318, 155 (1993).

[66] A. Djouadi and P. Gambino, Phys. Rev. Lett. 73, 2528 (1994); A. Djouadi, P. Gambino and B. A. Kniehl, Nucl. Phys. B 523, 17 (1998); B. A. Kniehl, Phys. Rev. D 53, 6477 (1996); C. Anastasiou, S. Buehler, E. Furlan, F. Herzog and A. Lazopoulos, Phys. Lett. B 702, 224 (2011); G. Passarino, C. Sturm and S. Uccirati, Phys. Lett. B 706, 195 (2011); A. Denner, S. Dittmaier, A. Mück, G. Passarino, M. Spira, C. Sturm, S. Uccirati and M. M. Weber, Eur. Phys. J. C 72, 1992 (2012); A. Djouadi and A. Lenz, Phys. Lett. B 715, 310 (2012).

[67] J. R. Andersen et al., Eur. Phys. J. Plus 126, 81 (2011); B. Bellazzini, C. Csaki, J. Hubisz, J. Serra and J. Terning, JHEP 1211, 003 (2012); D. B. Franzosi and R. Foadi, Phys. Rev. D 88, 015013 (2013).

[68] A. Alloul, B. Fuks and V. Sanz, arXiv:1310.5150 [hep-ph].

[69] U. Baur and E. W. N. Glover, Nucl. Phys. B 339, 38 (1990).

[70] G. Isidori, A. V. Manohar and M. Trott, Phys. Lett. B 728, 131 (2014); P. Artoisenet et al., JHEP 1311, 043 (2013); I. Anderson et al., Phys. Rev. D 89, 035007 (2014).

[71] G. Isidori, Y. Nir and G. Perez, Ann. Rev. Nucl. Part. Sci. 60, 355 (2010); G. Isidori, arXiv:1302.0661 [hep-ph]; R. Harnik, J. Kopp and J. Zupan, JHEP 1303, 026 (2013).

[72] J. M. Maldacena, Adv. Theor. Math. Phys. 2, 231 (1998).

[73] L. Randall and R. Sundrum, Phys. Rev. Lett. 83, 3370 (1999).

[74] C. Csaki, J. Hubisz and P. Meade, hep-ph/0510275.

[75] S. R. Coleman, J. Wess and B. Zumino, Phys. Rev. 177, 2239 (1969); C. G. Callan, Jr., S. R. Coleman, J. Wess and B. Zumino, Phys. Rev. 177, 2247 (1969).

[76] G. Buchalla and O. Cata, JHEP 1207, 101 (2012); G. Buchalla, O. Cata and C. Krause, Nucl. Phys. B 880, 552 (2014), and arXiv:1312.5624 [hep-ph].

[77] R. S. Gupta, H. Rzehak and J. D. Wells, Phys. Rev. D 86, 095001 (2012).

[78] O. Lebedev, H. M. Lee and Y. Mambrini, Phys. Lett. B 707, 570 (2012); A. Djouadi, O. Lebedev, Y. Mambrini and J. Quevillon, Phys. Lett. B 709, 65 (2012).

[79] LHC Higgs Cross Section Working Group, S. Dittmaier, C. Mariotti, G. Passarino, and R. Tanaka (Eds.), CERN-2011-002 (CERN, Geneva, 2011), [arXiv:1101.0593 [hep-ph]]; CERN-2012-002 (CERN, Geneva, 2012), [arXiv:1201.3084 [hep-ph]]; LHC Higgs Cross Section Working Group, S. Heinemeyer, C. Mariotti, G. Passarino, and R. Tanaka (Eds.), CERN-2013-004 (CERN, Geneva, 2013), [arXiv:1307.1347 [hep-ph]]; https: //twiki.cern.ch/twiki/bin/view/LHCPhysics/CrossSections.

[80] V. D. Barger, J. L. Hewett and R. J. N. Phillips, Phys. Rev. D 41, 3421 (1990).

[81] G. Aad et al. [ATLAS Collaboration], ATLAS-CONF-2012-160, ATLAS-CONF-2012-161, ATLAS-CONF-2013012, ATLAS-CONF-2013-013, ATLAS-CONF-2013-030; S. Chatrchyan et al. [CMS Collaboration], CMS-PASHIG-13-001, CMS-PAS-HIG-13-002, CMS-PAS-HIG-13-003, CMS-PAS-HIG-13-004, and Phys. Rev. D 89, 012003 (2014).

[82] A. Barroso, P. M. Ferreira, R. Santos, M. Sher and J. P. Silva, arXiv:1304.5225 [hep-ph]; B. Coleppa, F. Kling and S. Su, JHEP 1401, 161 (2014); O. Eberhardt, U. Nierste and M. Wiebusch, JHEP 1307, 118 (2013); N. Craig, 
J. Galloway and S. Thomas, arXiv:1305.2424 [hep-ph]; L. Wang and X.-F. Han, arXiv:1312.4759 [hep-ph]; A. Celis, V. Ilisie and A. Pich, JHEP 1312, 095 (2013); K. Cheung, J. S. Lee and P.-Y. Tseng, JHEP 1401, 085 (2014).

[83] For early studies see e.g. M. S. Carena, J. R. Espinosa, M. Quiros and C. E. M. Wagner, Phys. Lett. B 355, 209 (1995); H. E. Haber, R. Hempfling and A. H. Hoang, Z. Phys. C 75, 539 (1997); S. Heinemeyer, W. Hollik and G. Weiglein, Eur. Phys. J. C 9, 343 (1999); G. Degrassi, S. Heinemeyer, W. Hollik, P. Slavich and G. Weiglein, Eur. Phys. J. C 28, 133 (2003).

[84] M. S. Carena, H. E. Haber, H. E. Logan and S. Mrenna, Phys. Rev. D 65, 055005 (2002) [Erratum-ibid. D 65, $099902(2002)]$.

[85] P. Bechtle, S. Heinemeyer, O. Stål, T. Stefaniak, G. Weiglein and L. Zeune, Eur. Phys. J. C 73, 2354 (2013); G. Belanger, B. Dumont, U. Ellwanger, J. F. Gunion and S. Kraml, Phys. Rev. D 88, 075008 (2013); A. Arbey, M. Battaglia and F. Mahmoudi, Phys. Rev. D 88, 095001 (2013).

[86] M. S. Carena, H. E. Haber, H. E. Logan and S. Mrenna, Phys. Rev. D 65, 055005 (2002) [Erratum-ibid. D 65, $099902(2002)]$

[87] P. Fayet, Nucl. Phys. B90, 104 (1975); R. Barbieri, S. Ferrara, C. A. Savoy, Phys. Lett. B119, 343 (1982); M. Dine, W. Fischler, M. Srednicki, Phys. Lett. B104, 199 (1982); H. P. Nilles, M. Srednicki, D. Wyler, Phys. Lett. B120, 346 (1983); J. M. Frere, D. R. T. Jones, S. Raby, Nucl. Phys. B222, 11 (1983); J. P. Derendinger, C. A. Savoy, Nucl. Phys. B237, 307 (1984).

[88] J. R. Ellis, J. F. Gunion, H. E. Haber, L. Roszkowski, F. Zwirner, Phys. Rev. D39, 844 (1989); M. Drees, Int. J. Mod. Phys. A4, 3635 (1989); U. Ellwanger, M. Rausch de Traubenberg, C. A. Savoy, Phys. Lett. B315, 331 (1993); Z. Phys. C67, 665 (1995); Nucl. Phys. B492, 21 (1997); T. Elliott, S. F. King, P. L. White, Phys. Lett. B351, 213 (1995); S. F. King, P. L. White, Phys. Rev. D52, 4183 (1995); F. Franke, H. Fraas, Int. J. Mod. Phys. A12, 479 (1997).

[89] For reviews see, M. Maniatis, Int. J. Mod. Phys. A25, 3505 (2010); U. Ellwanger, C. Hugonie, A. M. Teixeira, Phys. Rept. 496, 1 (2010).

[90] R. D. Peccei and H. R. Quinn, Phys. Rev. Lett. 38, 1440 (1977); Phys. Rev. D16, 1791 (1977).

[91] S. F. King, M. Mühlleitner and R. Nevzorov, Nucl. Phys. B 860, 207 (2012); S. F. King, M. Mühlleitner, R. Nevzorov and K. Walz, Nucl. Phys. B 870, 323 (2013).

[92] U. Ellwanger, JHEP 1203, 044 (2012); K. Choi, S. H. Im, K. S. Jeong and M. Yamaguchi, JHEP 1302, 090 (2013); J.-J. Cao, Z.-X. Heng, J. M. Yang, Y.-M. Zhang and J.-Y. Zhu, JHEP 1203, 086 (2012); D. A. Vasquez, G. Belanger, C. Boehm, J. Da Silva, P. Richardson and C. Wymant, Phys. Rev. D 86, 035023 (2012); U. Ellwanger and C. Hugonie, Adv. High Energy Phys. 2012, 625389 (2012); K. Kowalska, S. Munir, L. Roszkowski, E. M. Sessolo, S. Trojanowski and Y.-L. S. Tsai, Phys. Rev. D 87, 115010 (2013); R. Barbieri, D. Buttazzo, K. Kannike, F. Sala and A. Tesi, Phys. Rev. D 87, 115018 (2013); D. T. Nhung, M. Mühlleitner, J. Streicher and K. Walz, JHEP 1311, 181 (2013); M. Badziak, M. Olechowski and S. Pokorski, JHEP 1306, 043 (2013); C. Beskidt, W. de Boer and D. I. Kazakov, Phys. Lett. B 726, 758 (2013).

[93] J. F. Gunion, Y. Jiang and S. Kraml, Phys. Rev. D 86, 071702 (2012); S. Munir, L. Roszkowski and S. Trojanowski, Phys. Rev. D 88, 055017 (2013).

[94] J. F. Gunion, Y. Jiang and S. Kraml, Phys. Rev. Lett. 110, 051801 (2013).

[95] J. R. Ellis, M. K. Gaillard and D. V. Nanopoulos, Nucl. Phys. B 106, 292 (1976); M. A. Shifman, A. I. Vainshtein, M. B. Voloshin and V. I. Zakharov, Sov. J. Nucl. Phys. 30, 711 (1979) [Yad. Fiz. 30, 1368 (1979)]; B. A. Kniehl and M. Spira, Z. Phys. C 69, 77 (1995).

[96] H. M. Georgi, S. L. Glashow, M. E. Machacek and D. V. Nanopoulos, Phys. Rev. Lett. 40, 692 (1978).

[97] M. Carena, I. Low and C. E. M. Wagner, JHEP 1208, 060 (2012).

[98] S. Dawson, A. Djouadi and M. Spira, Phys. Rev. Lett. 77, 16 (1996).

[99] T. Inami, T. Kubota and Y. Okada, Z. Phys. C 18, 69 (1983); A. Djouadi, M. Spira and P. M. Zerwas, Phys. Lett. B 264, 440 (1991); S. Dawson, Nucl. Phys. B 359, 283 (1991); M. Spira, A. Djouadi, D. Graudenz and P. M. Zerwas, Nucl. Phys. B 453, 17 (1995); K. G. Chetyrkin, B. A. Kniehl and M. Steinhauser, Phys. Rev. Lett. 79, 353 (1997); P. A. Baikov and K. G. Chetyrkin, Phys. Rev. Lett. 97, 061803 (2006).

[100] S. Gori and I. Low, JHEP 1309, 151 (2013).

[101] A. Azatov, R. Contino, A. Di Iura and J. Galloway, Phys. Rev. D 88, 075019 (2013).

[102] D. Lopez-Val, J. Sola and N. Bernal, Phys. Rev. D 81, 113005 (2010).

[103] C. Englert and M. McCullough, JHEP 1307, 168 (2013).

[104] N. Craig, C. Englert and M. McCullough, Phys. Rev. Lett. 111, 121803 (2013).

[105] A. Joglekar, P. Schwaller and C. E. M. Wagner, JHEP 1212, 064 (2012). 
[106] L. J. Hall, R. Rattazzi and U. Sarid, Phys. Rev. D 50, 7048 (1994); R. Hempfling, Phys. Rev. D 49, 6168 (1994); M. S. Carena, M. Olechowski, S. Pokorski and C. E. M. Wagner, Nucl. Phys. B 419, 213 (1994); D. M. Pierce, J. A. Bagger, K. T. Matchev and R.-J. Zhang, Nucl. Phys. B 491, 3 (1997); M. S. Carena, D. Garcia, U. Nierste and C. E. M. Wagner, Nucl. Phys. B 577, 88 (2000); G. D’Ambrosio, G. F. Giudice, G. Isidori and A. Strumia, Nucl. Phys. B 645, 155 (2002); A. J. Buras, P. H. Chankowski, J. Rosiek and L. Slawianowska, Nucl. Phys. B 659, 3 (2003); J. Guasch, P. Häfliger and M. Spira, Phys. Rev. D 68, 115001 (2003); V. Barger, H. E. Logan and G. Shaughnessy, Phys. Rev. D 79, 115018 (2009); N. D. Christensen, T. Han and S. Su, Phys. Rev. D 85, 115018 (2012).

[107] D. Noth and M. Spira, Phys. Rev. Lett. 101, 181801 (2008) and JHEP 1106, 084 (2011); L. Mihaila and C. Reisser, JHEP 1008, 021 (2010).

[108] For the corrections in the NMSSM see, J. Baglio, R. Gröber, M. Mühlleitner, D. T. Nhung, H. Rzehak, M. Spira, J. Streicher and K. Walz, arXiv:1312.4788 [hep-ph]. 